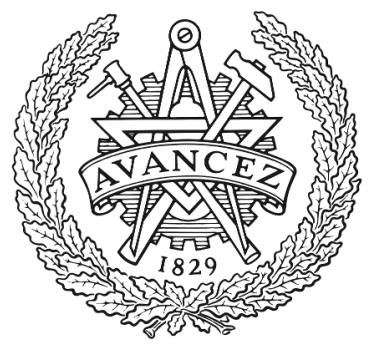

CHALMERS

UNIVERSITY OF TECHNOLOGY

\title{
Highly Stable Indacenodithieno[3,2-b]thiophene-Based Donor-Acceptor Copolymers for Hybrid Electrochromic and Energy Storage Applications
}

Downloaded from: https://research.chalmers.se, 2023-04-26 14:43 UTC

Citation for the original published paper (version of record):

Murto, P., Elmas, S., Mendez Romero, U. et al (2020). Highly Stable

Indacenodithieno[3,2-b]thiophene-Based Donor-Acceptor Copolymers for Hybrid

Electrochromic and Energy Storage Applications. Macromolecules, 53(24): 11106-11119.

http://dx.doi.org/10.1021/acs.macromol.0c02212

N.B. When citing this work, cite the original published paper. 


\title{
Highly Stable Indacenodithieno[3,2-b]thiophene-Based Donor- Acceptor Copolymers for Hybrid Electrochromic and Energy Storage Applications
}

\author{
Petri Murto, Sait Elmas, Ulises A. Méndez-Romero, Yanting Yin, Zewdneh Genene, Mariza Mone, \\ Gunther G. Andersson, Mats R. Andersson,* and Ergang Wang*
}

Cite This: Macromolecules 2020, 53, 11106-11119

Read Online

ACCESS | Lلlll Metrics \& More | 回 Article Recommendations | (1) Supporting Information

ABSTRACT: Stable doping of indacenodithieno[3,2-b] thiophene (IDTT) structures enables easy color tuning and significant improvement in the charge storage capacity of electrochromic polymers, making use of their full potential as electrochromic supercapacitors and in other emerging hybrid applications. Here, the IDTT structure is copolymerized with four different donoracceptor-donor (DAD) units, with subtle changes in their electron-donating and electron-withdrawing characters, so as to obtain four different donor-acceptor copolymers. The polymers attain important form factor requirements for electrochromic supercapacitors: desired switching between achromatic black and transparent states $\left(L^{*} a^{*} b^{*} 45.9,-3.1,-4.2 / 86.7,-2.2\right.$, and -2.7

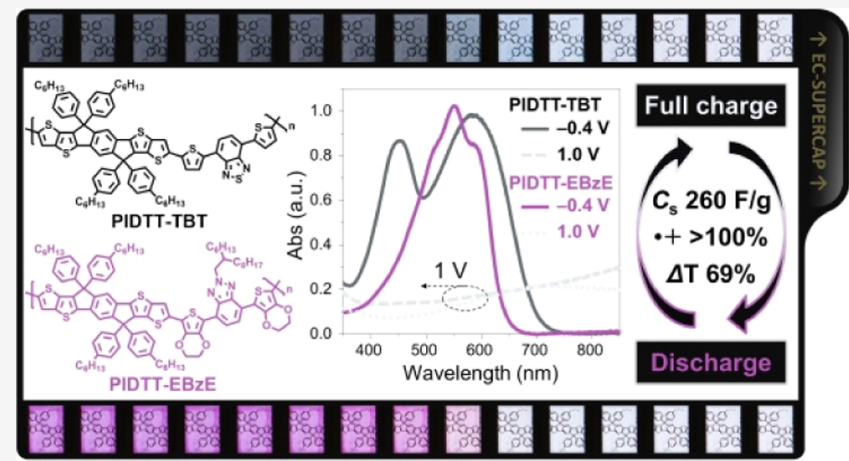
for PIDTT-TBT), high optical contrast ( $72 \%$ for PIDTTTBzT), and excellent electrochemical redox stability ( $\mathrm{I}^{\mathrm{red}} / \mathrm{I}^{\mathrm{ox}} c a .1 .0$ for PIDTT-EBE). Poly[indacenodithieno[3,2- $\left.b\right]$ thiophene-2,8diyl-alt-4,7-bis(2,3-dihydrothieno[3,4-b][1,4] dioxin-5-yl)-2-(2-hexyldecyl)-2H-benzo[d][1,2,3]triazole-7,7'-diyl] (PIDTT-EBzE) stands out as delivering simultaneously a high contrast $(69 \%)$ and doping level $(>100 \%)$ and specific capacitance $\left(260 \mathrm{~F} \mathrm{~g}^{-1}\right)$. This work introduces IDTT-based polymers as bifunctional electro-optical materials for potential use in color-tailored, colorindicating, and self-regulating smart energy systems.

\section{INTRODUCTION}

Fast-growing interest in electrochromic materials has resulted in a steady supply of conjugated polymers capable of changing color between two or more states, with the common feature of delivering reversible color change, high contrast, and stable long-term operation. ${ }^{1-5}$ This accordingly feeds the promised commercialization of smart windows/mirrors, ${ }^{6-8}$ eyewear, ${ }^{9}$ epaper, and other energy-efficient passive displays ${ }^{10,11}$ and their scale up by solution-based methods. ${ }^{12,13}$ On the other hand, conjugated polymers exhibit an intrinsic property of switching between conjugated and quinoidal forms upon electrochemical oxidation (p-type doping) and reduction (n-type doping), and this property has opened another interesting possibility to store energy through sufficient stabilization of the doped states. The concept of energy storage in conjugated polymers was introduced more than three decades ago by Heeger et al., ${ }^{14-16}$ and thereafter, plentiful research efforts have been focused on improving the charge storage capacity of the "pseudocapacitive" polymers for use as electrodes (or composite electrodes) in solid-state batteries and supercapacitors. ${ }^{17-19}$ An intriguing concept follows, as the electrochromic and energy storage properties of conjugated polymers can be merged into a single, bifunctional electro-optical material for use in electrochromic supercapacitors and other hybrid applications where the stored energy level can be visually observed or more precisely optically detected in real time. ${ }^{20}$ The switchable coloration can be tuned to transmit/block specific wavelengths in the visible and near-infrared (NIR) spectral regions for a particular interest in smart regulation of organic photovoltaics (OPVs), that is, integration of the two devices into self-powered systems that can simultaneously harvest and store solar energy. ${ }^{21}$ Recent work of Cho, et al. ${ }^{22}$ stands as an example of electrochromic supercapacitors based on a viologen/dimethyl ferrocene gel composite that was integrated with a semitransparent OPV device, effectively regulating the transmission at 545, 596, and $602 \mathrm{~nm}$ wavelengths when fully charged.

The vast majority of electrochromic polymers are based on 3,4-ethylenedioxythiophene (EDOT) and similar dioxythio-

Received: September 28, 2020

Revised: November 24, 2020

Published: December 11, 2020

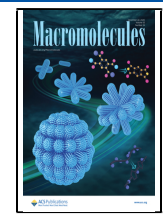


phene building blocks because of their low oxidation potential, fast color switching, and relatively simple chemical structures that allow easy color tuning, as spearheaded by the Reynolds group. $^{23-27}$ Other groups have developed similar structures to further tune the doping characteristics, optical contrast, and color purity of the polymers, for example, by means of chemical doping and electropolymerization. ${ }^{28-31}$ However, polymers based on the EDOT and dioxythiophene structures are often identified by a blue transmission window in their neutral state or remaining absorption band in the low-energy visible spectral region, causing a characteristic blue tint to the oxidized state. To counteract this issue, an inverse approach has been developed where the effective conjugation of the polymer backbone has been reduced to obtain a weakly absorbing neutral state, for example, by incorporating twisted triphenylamine and other arylamine building blocks, and a strongly absorbing colored state is obtained upon oxidation with an optical contrast exceeding $90 \%$. $^{32,33}$

Being designed mainly for fulfilling the optical requirements at minimal energy cost, the conventional electrochromic polymers exhibit relatively low charge storage capacities. Hence, completely different design approaches have been used to obtain conjugated polymers with sufficiently high specific capacitances $\left(C_{\mathrm{s}}\right)$ above $100 \mathrm{~F} \mathrm{~g}^{-1}$ (or volumetric capacitances in the order of $10^{5} \mathrm{mF} \mathrm{cm}^{-3}$ ). One example is a carbazole-based donor-acceptor copolymer, where the highest occupied molecular orbital (HOMO) and the lowest unoccupied molecular orbital (LUMO) were spatially separated. ${ }^{34}$ This action improved the electrochemical stability of the polymer during 2000 charge-discharge cycles, as compared to the corresponding polymer with the HOMO and LUMO delocalized along the conjugated backbone, but no connection to electrochromic functionality was reported. Polyanilines are common electrode materials in supercapacitors because of their high capacitances in the excess of $600 \mathrm{~F} \mathrm{~g}^{-1}$ (or $6 \times 10^{5} \mathrm{mF} \mathrm{cm}^{-3}$ ). ${ }^{35,36}$ Other examples include donor-acceptor copolymers incorporating naphthalene diimide and quinoxaline acceptors ${ }^{37,38}$ and microporous polymers exhibiting improved electrolyte ion transport and a high $C_{\mathrm{s}}$ of up to $576 \mathrm{~F} \mathrm{~g}^{-1}$ (for details about the experimental setups, refer to the cited literature). ${ }^{39,40}$

Chen, et al. ${ }^{41}$ reported a stretchable electrochromic supercapacitor based on a polyaniline/carbon nanotube composite electrode, which exhibited a high $C_{\mathrm{s}}$ of $308 \mathrm{~F} \mathrm{~g}^{-1}$ and visually observable color change. Poly(3,4-ethylenedioxythiophene) (PEDOT) derivatives are more conductive but tend to exhibit lower capacitances than the aforementioned examples because of their limited doping levels of $33 \%$ or so. ${ }^{42-44}$ A handful of examples exist where EDOT-based copolymers have been used in combined electrochromic and charge storage applications delivering either a high optical contrast of up to $75 \%$ or high capacitance of up to $92 \mathrm{~F} \mathrm{~g}^{-1}$ (linked to a lower contrast of $46 \%$ ) under applied potential switches. ${ }^{45-48}$ Crosslinking has been reported to stabilize a carbazole-benzodithiophene copolymer, maintaining its capacitance at $42 \mathrm{~F} \mathrm{~g}^{-1}$ after 5000 cycles, while the maximum optical contrast was $46 \%{ }^{49}$ Yun and co-workers ${ }^{50}$ obtained a much improved $C_{\mathrm{s}}$ of $471 \mathrm{~F} \mathrm{~g} \mathrm{~g}^{-1}$ and moderate contrast of $\mathrm{ca}$. $42 \%$ from a stretchable electrochromic supercapacitor using a $\mathrm{WO}_{3}$ nanotube/PEDOT:PSS composite electrode. Considering practical applications, it would be appealing to simplify the electrode design and develop polymers that can deliver both performance factors as a single active material in the solid state, that is, substantial color changes indicating high energy storage levels.

Conjugated polymers incorporating extended thiophenebased structures, such as the rigid and planar indacenodithiophene (IDT) and indacenodithieno[3,2- $b]$ thiophene (IDTT) backbones, are known for their high absorption coefficients and excellent electrochemical stabilities and charge transport kinetics, which have made them attractive for use as photoactive materials in OPVs, ${ }^{51-53}$ organic photodetectors (OPDs), ${ }^{54,55}$ organic field-effect transistors (OFETs), ${ }^{56,57}$ organic light-emitting diodes (OLEDs), ${ }^{58}$ and light-emitting electrochemical cells (LECs) ${ }^{59,60}$ and recently in electrochromic devices ${ }^{61,62}$ and hybrid electrochromic supercapacitors. ${ }^{63}$ However, significant improvements to the combined optical contrast and charge storage performance are needed for the realization of hybrid electrochromic and energy storage applications.

Herein, we synthesized four different donor-acceptordonor (DAD) units and copolymerized them with the IDTT donor monomer to obtain four different donor-acceptor copolymers, with a specific aim of introducing electrochemically stable alternatives to the EDOT-based polymers for use in all-polymer electrochromic supercapacitors. Together with the improved stability, the IDTT structure ensured strong optical absorption, while the varied $\mathrm{DAD}$ units delivered distinctively different optical absorption profiles and colors to the polymers. Despite the different colorations, all polymers featured similarly bleached transparent states without the obvious blue tint observed in EDOT-based polymers. The generally good electrochemical stability of the polymers is manifested by an uninterrupted operation over 1800 charge-discharge cycles while maintaining $80-98 \%$ of their initial optical contrast. Specifically, incorporation of thiophene as the donor and benzo $[c][1,2,5]$ thiadiazole as the acceptor in the DAD unit in PIDTT-TBT ensured fully reversible anodic oxidation and cathodic reduction with much desired electrochromic switching between achromatic gray/black and transparent states. The electrochemical reversibility in the anodic range was significantly improved using EDOT as the donor in PIDTT-EBE. The ratio between reduction and oxidation peak potentials $\left(I^{\mathrm{red}} / I^{\mathrm{ox}}\right)$ was close to $1.0 \mathrm{but}$, however, with a concomitant penalty of decreased optical contrast. The optical contrast was improved to $72 \%$ using thiophene as the donor and benzo $[d][1,2,3]$ triazole as the acceptor in PIDTT-TBzT. Further design using EDOT as the donor in PIDTT-EBzE combined the benefits of the polar polymer backbone and spacing of the polymer chains by alkyl side chains, which improved the electrolyte ion ingress and p-type doping in the excess of $100 \%$, thus maximizing the charge storage capacity. Nevertheless, the measured capacitances were substantially high for all four polymers, varying in the range of $1.3-2.3 \times$ $10^{5} \mathrm{mF} \mathrm{cm}^{-3}$ at the high scan rate of $100 \mathrm{mV} \mathrm{s}^{-1}$. The corresponding specific capacitances were evaluated to be 110$190 \mathrm{~F} \mathrm{~g}^{-1}$, which have not been achieved for other polymers containing similar DAD structures or for other charge-storing polymers in combination with high contrast optical switching, that is, without the IDTT structure in the polymer backbone. $^{45,46,48,64-66}$ Lower scan rates further improved the capacitance, eventually reaching $260 \mathrm{~F} \mathrm{~g}^{-1}$ at $5 \mathrm{mV} \mathrm{s}^{-1}$ in the case of PIDTT-EBzE. We envision that the combination of high-contrast electrochromic switching and high charge storage capacity opens new possibilities to unconventional 
Scheme 1. Chemical Structures of PIDTT-TBT, PIDTT-EBE, PIDTT-TBzT, and PIDTT-EBzE
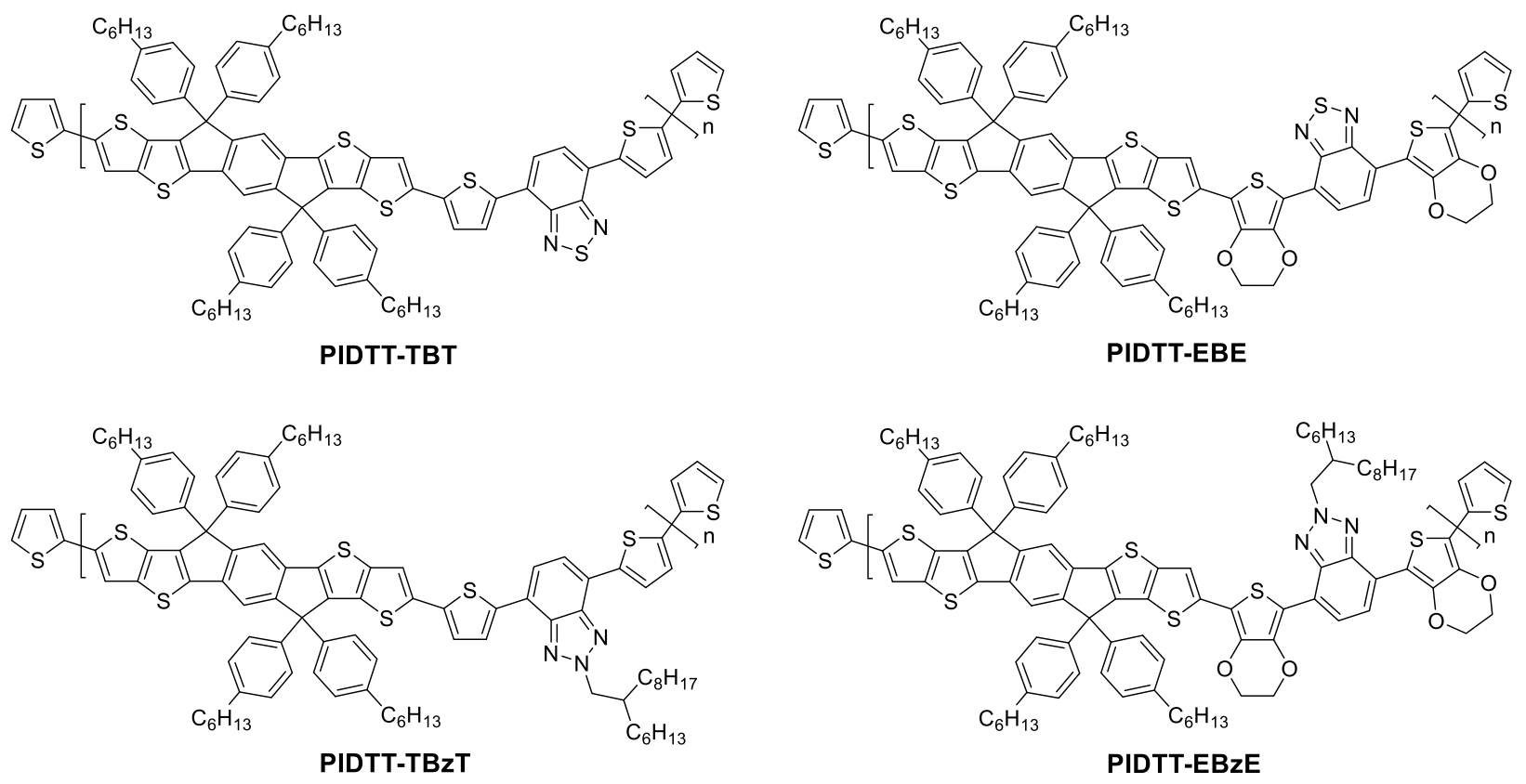

applications of these and other (published and unpublished) IDTT-based polymers.

\section{RESULTS AND DISCUSSION}

Chemical structures of the four copolymers PIDTT-TBT, PIDTT-EBE, PIDTT-TBzT, and PIDTT-EBzE are shown in Scheme 1, while the detailed synthetic procedures for the $\mathrm{DAD}$ segments and the copolymers are represented in Schemes S1 and S2 in the Supporting Information. For the design of the copolymers, we chose IDTT as an extended rigid donor group because IDTT-based polymers can deliver electrochromic switching at ultralow voltages down to $0-0.6$ $\mathrm{V}$ and thereby excellent stability upon electrochemical p- and n-type doping. ${ }^{62}$ The bulky hexylphenyl groups were selected as solubilizing substituents with the prospect of facilitating scalable processing. Different combinations of the electron-rich EDOT donor monomer and either of the electron-deficient benzo $[c][1,2,5]$ thiadiazole or benzo $[d][1,2,3]$ triazole acceptor monomers are commonly used in well-functioning electrochromic polymers. ${ }^{26,28,64,67}$ These two acceptor moieties are also present in some charge storage polymers. ${ }^{46,48,65,66}$ Chemical coupling of the donor and acceptor units (so-called electron "push-pull" design) allows easy tuning of the energy gap $\left(E_{\mathrm{g}}\right)$, oxidation potential, color, and electrochromic switching kinetics of the polymers. We introduced less-polar thiophene as an alternative to the highly polar EDOT donor and used the abovementioned building blocks to construct four different $\mathrm{DAD}$ structures with varying optical and electrical properties: either based on the stronger electron acceptor in 4,7-bis (5-bromothiophen-2-yl)benzo[ $c][1,2,5]$ thiadiazole (TBT) and 4,7-bis(7-bromo-2,3-dihydrothieno[3,4-b][1,4]dioxin-5-yl)benzo[c] $[1,2,5]$ thiadiazole (EBE) monomers or the weaker electron acceptor in 4,7-bis (5-bromothiophen-2yl)-2-(2-hexyldecyl)-2H-benzo[ $d][1,2,3]$ triazole (TBzT) and 4,7-bis (7-bromo-2,3-dihydrothieno[3,4-b][1,4] dioxin-5-yl)-2(2-hexyldecyl)-2H-benzo[d][1,2,3]triazole (EBzE) monomers. The 2-hexyldecyl side chain was introduced to the latter two $\mathrm{DAD}$ structures for improved solubility.

Each of the dibromo-substituted TBT, EBE, TBzT, and EBzE monomers were copolymerized with the bis(trimethylstannyl)-substituted IDTT monomer via Pd-catalyzed Stille polycondensation. Good degrees of polymerization were observed for PIDTT-TBT and PIDTT-TBzT, with number-average molecular weights $\left(M_{\mathrm{n}}\right)$ of 24.8 and $72.8 \mathrm{~kg}$ $\mathrm{mol}^{-1}$, respectively (Figure S1, Supporting Information). PIDTT-TBzT presents improved solubility and higher $M_{n}$ because of the 2-hexyldecyl side chains. However, the molecular weights of PIDTT-EBE and PIDTT-EBzE cannot be determined using the gel permeation chromatography (GPC) technique, which we attribute to the highly polar EDOT groups in these two polymer backbones and their adhesive interaction with the stationary phase. Similar issues were not observed when the EDOT concentration was decreased in the IDTT backbone. ${ }^{59}$ Matrix-assisted laser desorption ionization-time of flight (MALDI-TOF) mass spectrometry requires evaporation of the ionized material in high vacuum, which is practical for the TBT, EBE, TBzT, and EBzE monomers and short-chain oligomers with an $M_{n}$ of around $10 \mathrm{~kg} \mathrm{~mol}^{-1}$ or less, ${ }^{68,69}$ and our MALDI-TOF experiments accordingly suggest that PIDTT-EBE and PIDTT-EBzE exhibit polymeric chain lengths because of the absence of oligomer-sized fractions. We confirmed the chemical structures and compositions of all four polymers using ${ }^{1} \mathrm{H}$ NMR spectroscopy and elemental analysis techniques (see Figures S2 and S3, Supporting Information). The chemical shifts of the polymers are designated to those of the IDTT and either the TBT, EBE, TBzT, or EBzE donoracceptor repeating units of the PIDTT-TBT, PIDTT-EBE, PIDTT-TBzT, and PIDTT-EBzE copolymers, respectively. Three of the polymers, PIDTT-TBT, PIDTT-TBzT, and PIDTT-EBzE, were isolated at high yields of $c a .90 \%$, while the lower yield of PIDTT-EBE (34\%) is attributed to the formation of insoluble, presumably very high $M_{\mathrm{n}}$ polymer fraction in the polycondensation. Importantly, however, the isolated polymer fractions were soluble and processable either from concentrated chlorobenzene solutions $\left(10-20 \mathrm{mg} \mathrm{mL}^{-1}\right)$ or dilute toluene solutions $\left(1-2 \mathrm{mg} \mathrm{mL}^{-1}\right)$. Thermogravi- 

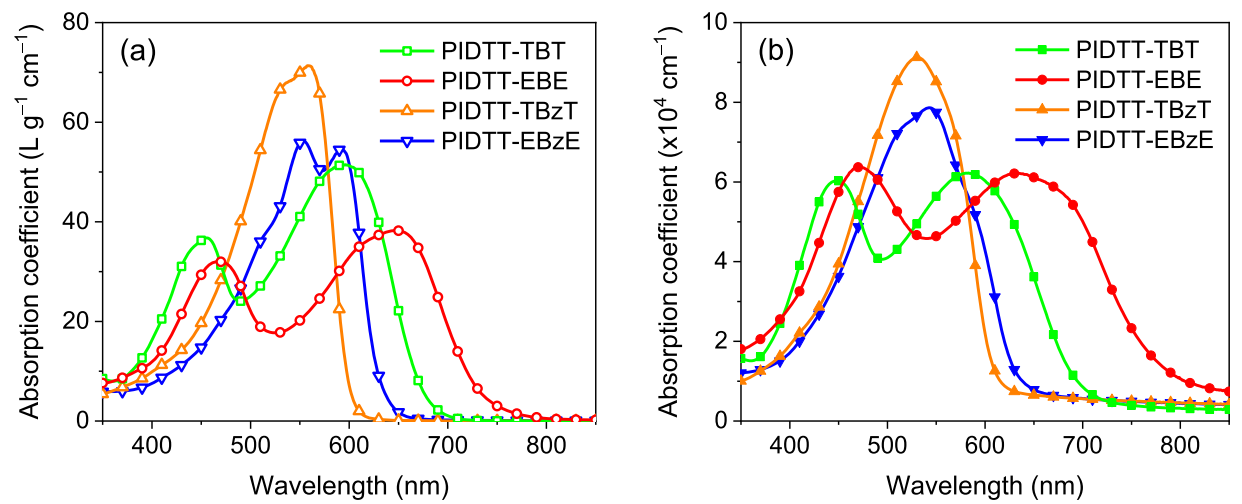

Figure 1. Absorption coefficient of the polymers (a) in chlorobenzene solution and (b) as a solid-state thin film. The thin-film samples were prepared by spin coating onto a glass substrate.

metric analysis (TGA) of the polymers shows that they all feature excellent thermal stabilities with $1 \%$ weight loss occurring at high temperatures of $T_{99}>390{ }^{\circ} \mathrm{C}$ (Figure S4, Supporting Information). The lack of thermal transitions in differential scanning calorimetry (DSC) measurements indicates that none of the polymers exhibit significant crystallinity (Figure S5, Supporting Information), which would inhibit the electrolyte ion ingress during the electrochromic operation.

Figure 1 shows the absorption coefficient versus wavelength curves of the polymers in chlorobenzene solution and as a solid-state thin film. PIDTT-TBT and PIDTT-EBE featured a characteristic two-band absorption profile of a donoracceptor copolymer, which we tentatively attribute to lowenergy intramolecular charge transfer (ICT) transitions and high-energy local excitations in the donor-rich polymer backbone. The general observation is that the high-energy peak shifted toward a longer wavelength and its intensity increased relative to the ICT band when going from PIDTTTBT to PIDTT-EBE, in accordance with an increasing electron-rich character of the polymer. In solution, PIDTTTBT exhibited a stronger ICT band peaking at $596 \mathrm{~nm}$ as compared to the corresponding absorption of PIDTT-EBE at $649 \mathrm{~nm}$. In the solid state, the two absorption bands became more balanced as a result of increasing $\pi-\pi$ interactions, that is, stacking of the polymer chains. However, the dominant ICT band of PIDTT-TBT is attributed to weaker electron donor contribution of the two thiophene spacers as compared to PIDTT-EBE where the acceptor is endowed with two EDOT spacers. The same trend is observed in both solution and thinfilm samples. This stems from our design motif, as the more balanced push-pull character of the polymer is anticipated to induce more effective formation of intrachain charge-transfer states along the PIDTT-TBT donor-acceptor backbone. ${ }^{70}$ We further note that the onset of absorption of PIDTT-EBE is red-shifted over $50 \mathrm{~nm}$ (and only half of that for PIDTTTBT) when going from solution to thin films, while the highenergy peak position remained essentially unchanged for both polymers. This translates to a substantial broadening of the full width at half maximum (fwhm) in the solid state $(257 \mathrm{~nm}$ for PIDTT-TBT and $324 \mathrm{~nm}$ for PIDTT-EBE). Optical band gaps $\left(E_{\text {opt }}\right)$ of 1.77 and $1.58 \mathrm{eV}$ were estimated for PIDTTTBT and PIDTT-EBE, respectively, from the low-energy onsets of absorption in thin films.

The other two polymers, PIDTT-TBzT and PIDTT$\mathrm{EBzE}$, featured distinctively different absorption envelopes. In both cases, the local excitations and ICT transitions are observed to merge as a narrow, energetically overlapping single band with a peak maximum at $558 \mathrm{~nm}$ (for PIDTT-TBzT) and $553 \mathrm{~nm}$ (for PIDTT-EBzE) in solution, but the absorption of PIDTT-EBzE was more structured. Strong single-band absorption is a typical characteristic of a polymer containing only electron-rich units such as the PIDTT homopolymer. ${ }^{62,71} \mathrm{We}$ note that the strong and narrow absorption of these two polymers is retained in the solid state, with the difference that the PIDTT-TBzT band was more redshifted upon transition from solution to thin films. We attribute these features to the weak electron acceptor that accounts for a weak charge-transfer interaction and a dominant donor contribution in the optical absorption. Another feature that differentiates the PIDTT-TBzT/PIDTT-EBzE pair from the PIDTT-TBT/PIDTT-EBE pair is that the thin-film onset of absorption was not significantly red-shifted when replacing the thiophene spacers in PIDTT-TBzT with the EDOT spacers in PIDTT-EBzE. As a result, the fwhm remained narrow for both PIDTT-TBzT $(128 \mathrm{~nm})$ and PIDTT-EBzE $(148 \mathrm{~nm})$ in the solid state and an $E_{\text {opt }}$ of 2.03 and $1.95 \mathrm{eV}$ were estimated for the two polymers, respectively. PIDTT-TBzT featured the narrowest absorption band in this series, in both solution and thin films, which in turn translates to the highest absorption coefficient because of the coalescence of energetically overlapping optical transitions.

To predict the potential of PIDTT-TBT, PIDTT-TBzT, PIDTT-EBE, and PIDTT-EBzE for p-doping, we carried out density functional theory (DFT) calculations for neutral and oxidized polymers. Two repeating unit model structures were used to identify the oxidation sites in the polymers (see Figures S6-S10, Supporting Information). In the case of the dimers, one and two electron oxidations correspond to 50 and $100 \%$ doping, respectively. The thiophene-containing polymers PIDTT-TBT and PIDTT-TBzT are effectively planarized upon oxidation. On the other hand, the EDOT-containing PIDTT-EBE and PIDTT-EBzE feature coplanar conformations also in their neutral states, and oxidation does not cause any backbone distortion either. The structural integrity of the latter two polymers is highly favorable with regard to electrochemical stability during the charge-discharge cycles. Comparing the doping of the PIDTT-TBT/PIDTT-EBE pair, in PIDTT-TBT, the positive charge is localized at the IDTT donor units, whereas in PIDTT-EBE, the EDOT groups provide additional stabilization by delocalizing the charge. The same applies to the PIDTT-TBzT/PIDTT- 

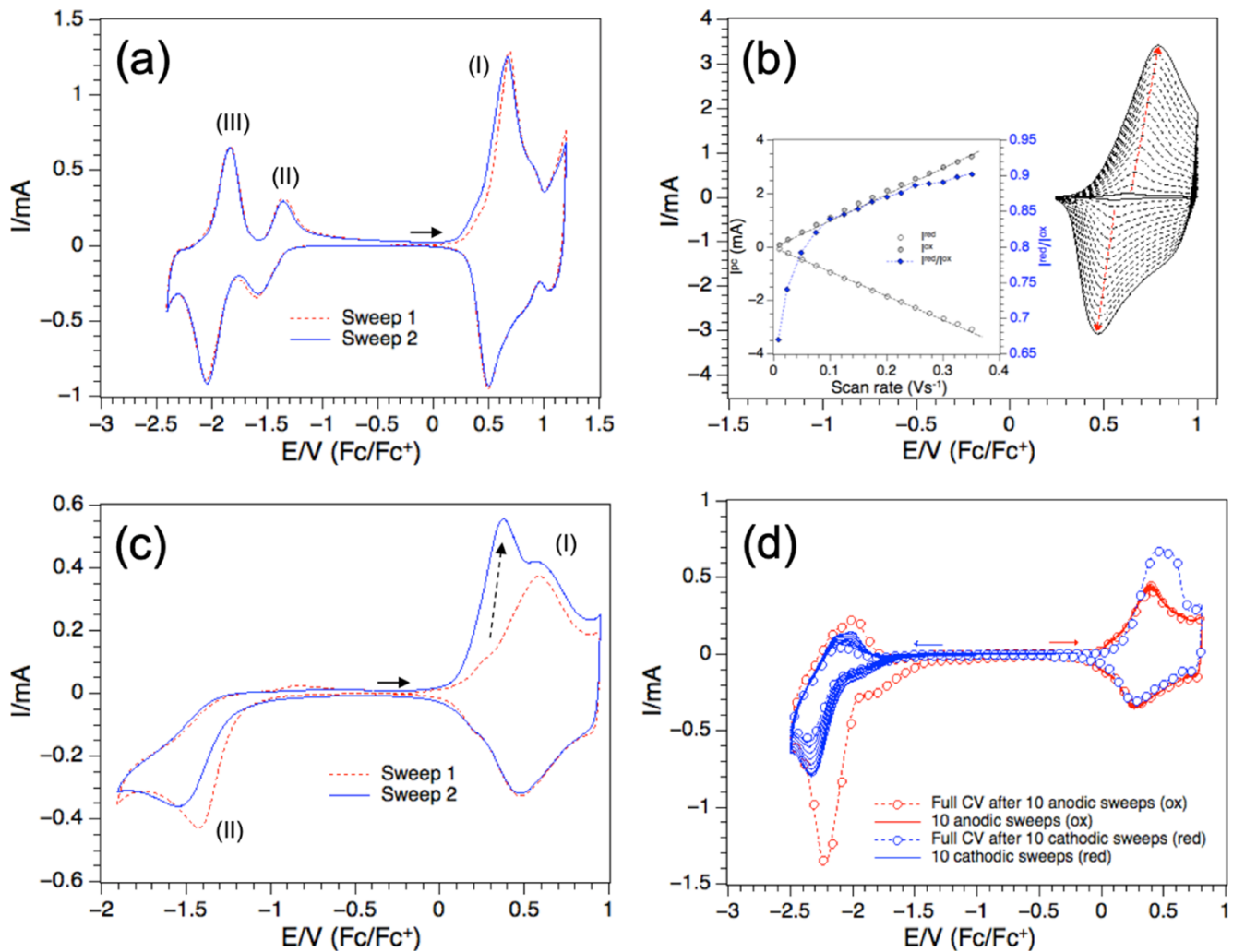

Figure 2. (a) Full cyclic voltammetry traces of PIDTT-TBT recorded at $100 \mathrm{mV} \mathrm{s}^{-1}$ and (b) its anodic response at different scan rates ranging from 10 to $350 \mathrm{mV} \mathrm{s}^{-1}$. The inset in (b) shows the peak currents $I^{\text {red }}$ and $I^{\text {ox }}$ together with the dedoping/doping ratio $\left(I^{\text {red }} / I^{\text {ox }}\right)$. (c) Full cyclic voltammetry traces of PIDTT-EBE at $100 \mathrm{mV} \mathrm{s}^{-1}$. (d) Charge-trapping effect of PIDTT-EBzE during electrochemical cycling in the anodic (red lines) and cathodic range (blue lines), as recorded at $100 \mathrm{mV} \mathrm{s}{ }^{-1}$. The supporting electrolyte was $0.1 \mathrm{M}$ solution of $\mathrm{NBu}_{4} \mathrm{PF}_{6}$ in anhydrous acetonitrile.

EBzE pair, with a notion that the oxidized triazole-ammonium group shares the positive charge, and thereby, in PIDTT$\mathrm{EBzE}$, the charge is even more delocalized in the donor and acceptor units over the entire polymer backbone. The effective charge delocalization is anticipated to lower the oxidation potential and facilitate high redox activity in the EDOTcontaining polymers, particularly in PIDTT-EBzE.

Electrochemical studies with assistance of X-ray photoelectron spectroscopy (XPS) surface analysis were conducted on PIDTT-TBT, PIDTT-EBE, PIDTT-TBzT, and PIDTT-EBzE films to understand the $\mathrm{p}$ - and n-type doping/dedoping cyclability and location of the generated charges in the synthesized polymers during electrochromic operation. Considering n-doping, the strong benzo[c] $[1,2,5]$ thiadiazole acceptor stands out as the most stabilizing unit in the polymer backbone. Unlike other IDTT-based polymers, PIDTT-TBT exhibits two distinct and reversible reduction peaks at $-1.47 \mathrm{~V}$ (II) and $-1.94 \mathrm{~V}$ (III) versus the ferrocene/ ferrocenium $\left(\mathrm{Fc} / \mathrm{Fc}^{+}\right)$redox couple (Figure $2 \mathrm{a}$ ), with the main reduction peak (III) originating from the IDTT donor moiety. ${ }^{72}$ The less-intense peak (II) at a lower potential is, therefore, ascribed to the reduction of the benzo[c] $[1,2,5]$ thiadiazole acceptor unit. The anodic oxidation (p-doping) peak of PIDTT-TBT appears reversible and sharp at $0.59 \mathrm{~V}$ (I) versus $\mathrm{Fc} / \mathrm{Fc}^{+}$. Notably, PIDTT-TBT did not suffer loss of either cathodic or anodic reversibility during full cyclic voltammetry $(\mathrm{CV})$ sweeps in the wide potential range between -2.5 and $1.2 \mathrm{~V}$ (i.e., only limited by the potential window of the solvent), as indicated by the unchanged peak (I-III) currents and their respective positions after two subsequent scans. This is an important example of a new line of electrochemically stable polymers that do not require protection of the 3,4-positions of the thiophene units, unless specifically intended, for example, for color-tuning purposes.

When introducing EDOT into the IDTT structure in PIDTT-EBE, the reversible oxidation peak is shifted down to $0.53 \mathrm{~V}$ (I) with the cathodic reduction appearing irreversible at $-1.5 \mathrm{~V}$ (II) (Figure 2c). As per definition, the reduction is interpreted as an irreversible process. However, the most interesting electrochemical feature of PIDTT-EBE is the electrochemical dedoping of the n-doped (reduced) film occurring in the anodic range, observed as an increased intensity of the peak at $0.37 \mathrm{~V}$ in the subsequent scan (Figure $2 c$, sweep 2). This effect is understood as a capacitive effect where charges are being trapped in the polymer structure during electrochemical cycling between different doping states. ${ }^{34}$ We speculate that changes in the applied electric field cause changes in the permeability of the deposited films for sufficient electrolyte ion ingress and release, effectively stabilizing the trapped charges. ${ }^{73,74}$ Unlike in PIDTT-TBT, the cathodic reversibility in PIDTT-EBE does not occur during the reverse scan; it requires a counter potential to be applied in the anodic range to extract the injected charges and to achieve a neutral state. This observation clearly highlights the importance of understanding the electrochemical redox processes of newly synthesized polymers, particularly when estimating their HOMO/LUMO energy levels from freshly deposited versus preconditioned films.

The tendency of the IDTT structures to accumulate charges is clearly highlighted in PIDTT-EBzE. When cycling PIDTT- 

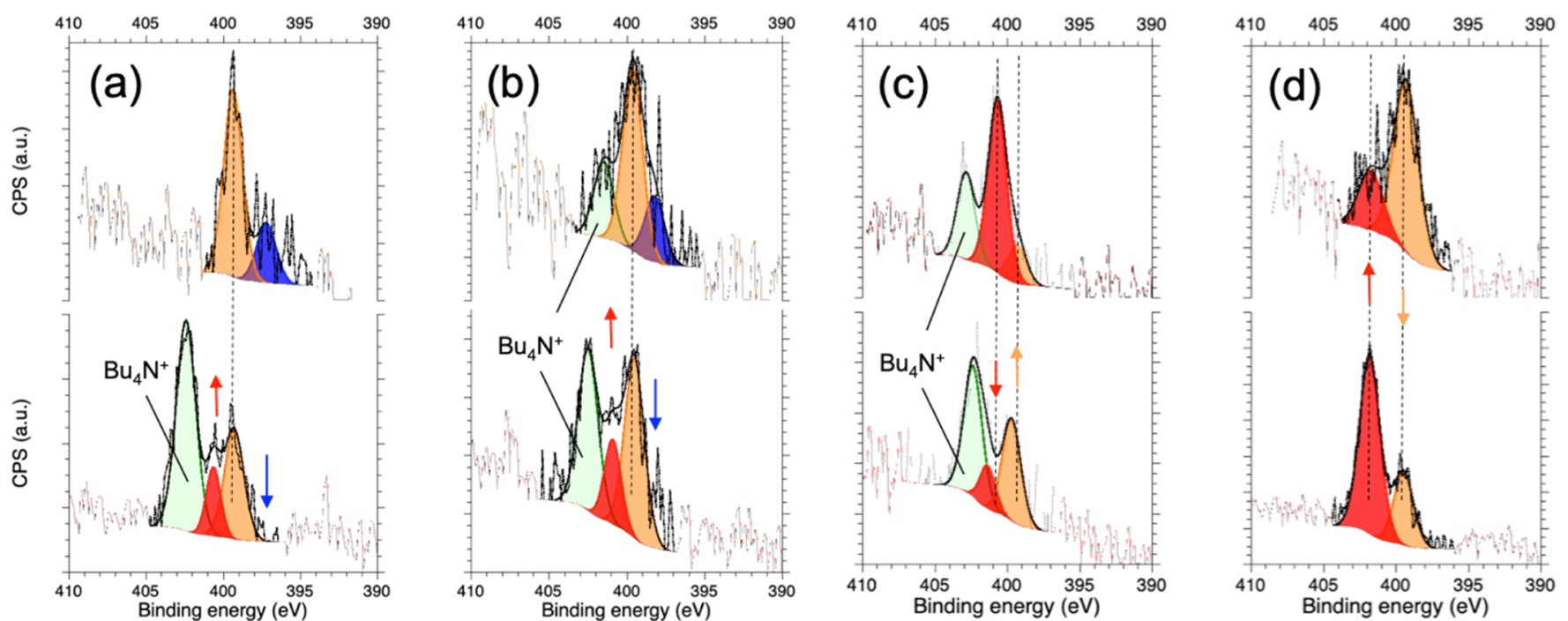

Figure 3. Fitted N(1s) XPS core-level spectra of (a) PIDTT-TBT, (b) PIDTT-EBE, (c) PIDTT-TBzT, and (d) PIDTT-EBzE in their (top panels) discharged neutral and (bottom panels) charged oxidized states, representing a complete charge-discharge cycle. The peaks are assigned to the oxidized state of the $\mathrm{C}=\mathrm{N}-\mathrm{S} / \mathrm{N}$ species (orange fit), its reduced version (blue fit), and further oxidized state of $\mathrm{C}=\mathrm{N}-\mathrm{S} / \mathrm{N}$ (red fit) in the acceptor unit of each polymer (see Table S1, Supporting Information, and detailed discussion therein). Quaternary nitrogen (green fit) is assigned to unbound $\mathrm{NBu}_{4} \mathrm{PF}_{6}$ electrolyte. The arrows indicate the change in intensity of the respective peaks upon oxidation (up: increase; down: decrease). The dotted lines are guides to the eye only and centered at the peaks of the neutral samples.

EBzE for 10 consecutive steps between neutral and p-doped states at $100 \mathrm{mV} \mathrm{s}^{-1}$ and sweeping the polymer thereafter in the full $\mathrm{CV}$ range, a gain in cathodic current by $70 \%$ was recorded (Figure $2 \mathrm{~d}$, red $\mathrm{CV}$ traces). When inversing the procedure and first cycling the same film in the cathodic range, the increase in anodic current goes up to $55 \%$ (Figure $2 \mathrm{~d}$, blue $\mathrm{CV}$ traces). We attribute the high redox activity of PIDTT$\mathrm{EBzE}$ to the effective charge delocalization/stabilization in the polymer backbone (see the DFT discussion mentioned above) and enhanced electrolyte ion ingress because of separation of the polar polymer chains by the 2-hexyldecyl side chains. The better electrolyte transport is directly observed as improved reversibility of the cathodic reduction of PIDTT-EBzE (Figure 2d), as compared to that of PIDTT-EBE (Figure 2c). PIDTT-TBT, PIDTT-EBE, and PIDTT-EBzE show linear peak currents during forward and reverse scans ( $I^{\mathrm{ox}}$ and $I^{\text {red }}$, respectively) with intensity ratios $\left(I^{\text {red }} / I^{\text {ox }}\right)$ close to 1.0 at fast scan rates $\geq 100 \mathrm{mV} \mathrm{s}^{-1}$ (Figures $2 \mathrm{~b}$ and S11, Supporting Information). At lower scan rates and controlled diffusion of the electrolyte, the relative peak ratios drop sharp toward 0.5, indicating stable charge trapping. PIDTT-TBzT appears as the least reversible candidate during electrochemical studies. The electrochemical stability increases in the order PIDTTTBzT « PIDTT-EBzE < PIDTT-TBT < PIDTT-EBE (with electrochemical stability, we refer to doping/dedoping cyclability of the polymer). This tendency is also reflected in the short-term CV cycling stability studies shown in Figure S12 (Supporting Information).

To trace the trapped charges during p-doping at low scan rates, the polymers were drop-casted on a platinum mesh each, oxidized for $3 \mathrm{~min}$ by halting the $\mathrm{CV}$ potential after passing the respective anodic peak, washed thoroughly with acetonitrile to remove the unbound background electrolyte, dried, and subjected to surface analysis using XPS (see the Supporting Information for details). The neutral films were prepared in the same way and used as reference compounds by passing the anodic $\mathrm{CV}$ scans and stopped at $-0.4 \mathrm{~V}$ versus $\mathrm{Fc} / \mathrm{Fc}^{+}$, representing a complete charge-discharge cycle. Figure 3 shows fitted $\mathrm{N}(1 \mathrm{~s})$ core-level spectra of PIDTT-TBT, PIDTT-EBE, PIDTT-TBzT, and PIDTT-EBzE after pdoping (bottom panels) compared with their neutral states after dedoping of the doped films (top panels). The corresponding $S(2 p)$ core-level spectra are included in Figure S13 (Supporting Information), while detailed assignments of the peak positions for $\mathrm{N}(1 \mathrm{~s}), \mathrm{S}(2 \mathrm{p}), \mathrm{O}(1 \mathrm{~s})$, and $\mathrm{C}(1 \mathrm{~s})$ are discussed in the Supporting Information and summarized in Table S1.

Overall, the fitted N(1s) core-level spectra of the PIDTTTBT/PIDTT-EBE pair are shifted toward higher binding energies in their oxidized states, highlighting the strong electron-deficient character of the benzo[ $c][1,2,5]$ thiadiazole acceptor, whereas their $S(2 p), O(1 s)$, and $C(1 s)$ core-level spectra slightly fluctuate, by less than $\pm 0.3 \mathrm{eV}$ (see Table S1, Supporting Information). Of particular note is the strong shift of $\mathrm{N}$ toward higher binding energies by 3.4 and $2.7 \mathrm{eV}$ in $\mathrm{C}=$ $\mathrm{N}-\mathrm{S}$ species in the acceptor unit in PIDTT-TBT and PIDTT-EBE, respectively, after $3 \mathrm{~min}$ of electrochemical oxidation, suggesting their transition from neutral states to higher levels of doped states (see the orange and red peaks in Figure $3 a, b){ }^{75}$ Such cationic doping is much stronger in contrast to the weak benzo $[d][1,2,3]$ triazole acceptor-containing PIDTT-TBzT/PIDTT-EBzE pair. PIDTT-TBzT was found as the least reversible candidate during the electrochemical stability tests and anodic oxidation at variable scan rates, as discussed earlier (see Figures S11a,b and S12c, Supporting Information). XPS analysis of its oxidized state revealed moderate shifts of the imine and amine functional groups up to $0.7 \mathrm{eV}$ toward higher binding energies but also showed strongest shifts of the $\mathrm{S}(2 \mathrm{p})$ core level spectra toward lower binding energies as compared to the other three polymers, indicating an electronic imbalance in the doped polymer backbone (see Figure S13 and Table S1, Supporting Information). In the case of PIDTT-EBzE, the electrochemical doping was less localized at the nitrogen atoms in the 

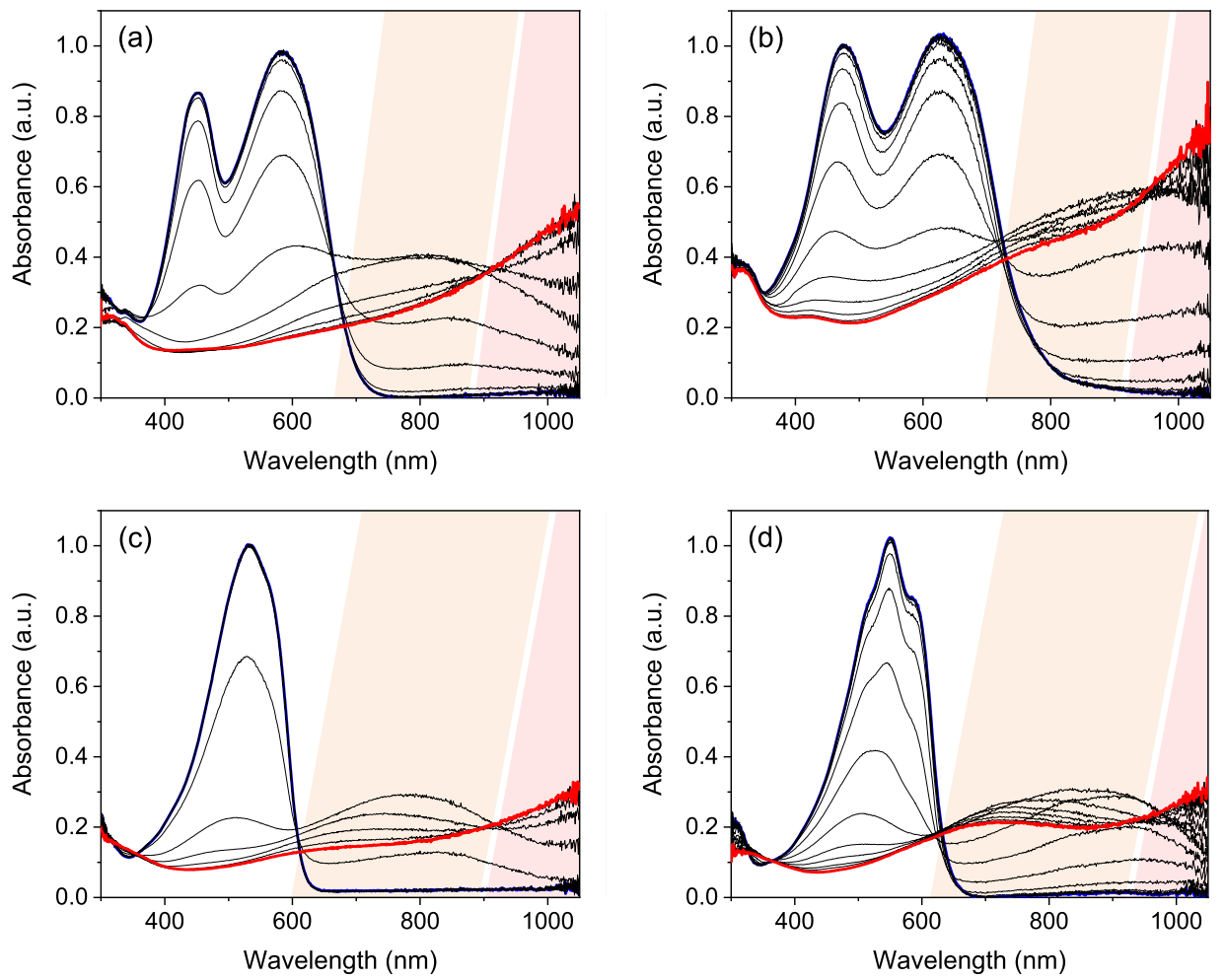

Figure 4. Electrochromic spectra of (a) PIDTT-TBT, (b) PIDTT-EBE, (c) PIDTT-TBzT, and (d) PIDTT-EBzE thin films, as prepared by spin coating onto an ITO/glass substrate. The spectra were measured by increasing the voltage from $-0.4 \mathrm{~V}$ (blue line) to $1.0 \mathrm{~V}$ (red line) vs Fc/ $\mathrm{Fc}^{+}$at intervals of $0.1 \mathrm{~V}$. The supporting electrolyte was $0.1 \mathrm{M}$ solution of $\mathrm{NBu}_{4} \mathrm{PF}_{6}$ in anhydrous acetonitrile. The polaronic and bipolaronic absorption regions are schematically illustrated by the orange and red highlights, respectively. The corresponding $L^{*} a^{*} b^{*}$ color coordinates are included in Figure S15 and Table S3 (Supporting Information), while Figure S16 (Supporting Information) shows the transmission spectra and photographs illustrating the development of the color of the films.

acceptor unit but instead more balanced by the EDOT groups as indicated in the positive shifts of the fitted binding energies of $\mathrm{O}(1 \mathrm{~s})$ in Table S1 (Supporting Information). In other words, the charge is evenly delocalized along the polymer backbone, effectively stabilizing the doped states, which was also suggested by the DFT calculations discussed earlier (i.e., also without taking into account the interactions of doped polymers and electrolyte counterions and following additional stabilization, which would be computationally very expensive to simulate).

Although the oxidized and neutral films on the Pt mesh were thoroughly washed with acetonitrile, XPS analysis detected unbound electrolyte salt $\left(\mathrm{NBu}_{4} \mathrm{PF}_{6}\right)$ as indicated by the $\mathrm{N}(1 \mathrm{~s})$ core-level spectra in Figure 3 and Table S1 (Supporting Information). Excluding neutral PIDTT-TBT, quaternary nitrogen was found in all other polymer samples both in their oxidized and neutral states, as indicated by the green peaks in Figure 3. As for PIDTT-EBzE, no indication for quaternary nitrogen can be made because of the strong overlap of triazole-ammonium species in the polymer structure.

High doping levels were calculated for the oxidized polymers based on the atomic compositions of the XPS samples in Table S2 (Supporting Information). PIDTT-TBT and PIDTT-EBE featured 82 and $62 \%$ p-doping after 3 min of electrochemical oxidation, which is equivalent to four out of five and three out of five repeating units being doped, respectively, whereas PIDTT-TBzT and PIDTT-EBzE exhibited $>100 \%$ doping levels upon oxidation. Significant percentage of p-doping was also found in the dedoped neutral state of PIDTT-EBE and to smaller extents in the neutral states of PIDTT-TBT and
PIDTT-TBzT, further indicating the inherent property of IDTT-based polymers for stable capacitive trapping of charges at low scan rates, in line with our earlier observation in $\mathrm{CV}$ measurements. PIDTT-EBzE was an exception in that it was the only polymer displaying complete dedoping in its neutral state, which is combined with highest doping levels in this study. We note, however, that the calculated doping levels only represent the top layer of the films because the penetration depth of XPS is within the few-nanometer range. During electrochemical cycling (doping/dedoping), the bottom layers of the films in close contact with the Pt electrode are effectively neutralized, becoming less conducting and insulating the doped polymer layers further away from the electrode. Oxidized structures of the neutral films (Figure 3, top panels) were detected under XPS because of the charge trapping in the top layers of the films. It is also noteworthy that the electrochemically conditioned films were stable in their neutral and oxidized states, as they were washed, dried, and stored under ambient conditions in total $3+$ days before they were subjected to XPS analysis (see the Supporting Information for details). The ability of the IDTT-based polymers to trap and stabilize charges highlights their potential as highly energy efficient materials for electrochromic operation and energy storage applications. Herein, no continuous input of energy is needed to maintain the doped states.

For the electrochromic characterization, the polymer films were either spin-coated or spray-coated onto an ITO-glass substrate, which functioned as the electrochemical working electrode. The polymers were coated to an absorbance of 1.00 \pm 0.03 a.u. to allow fair comparison of their optical properties, 

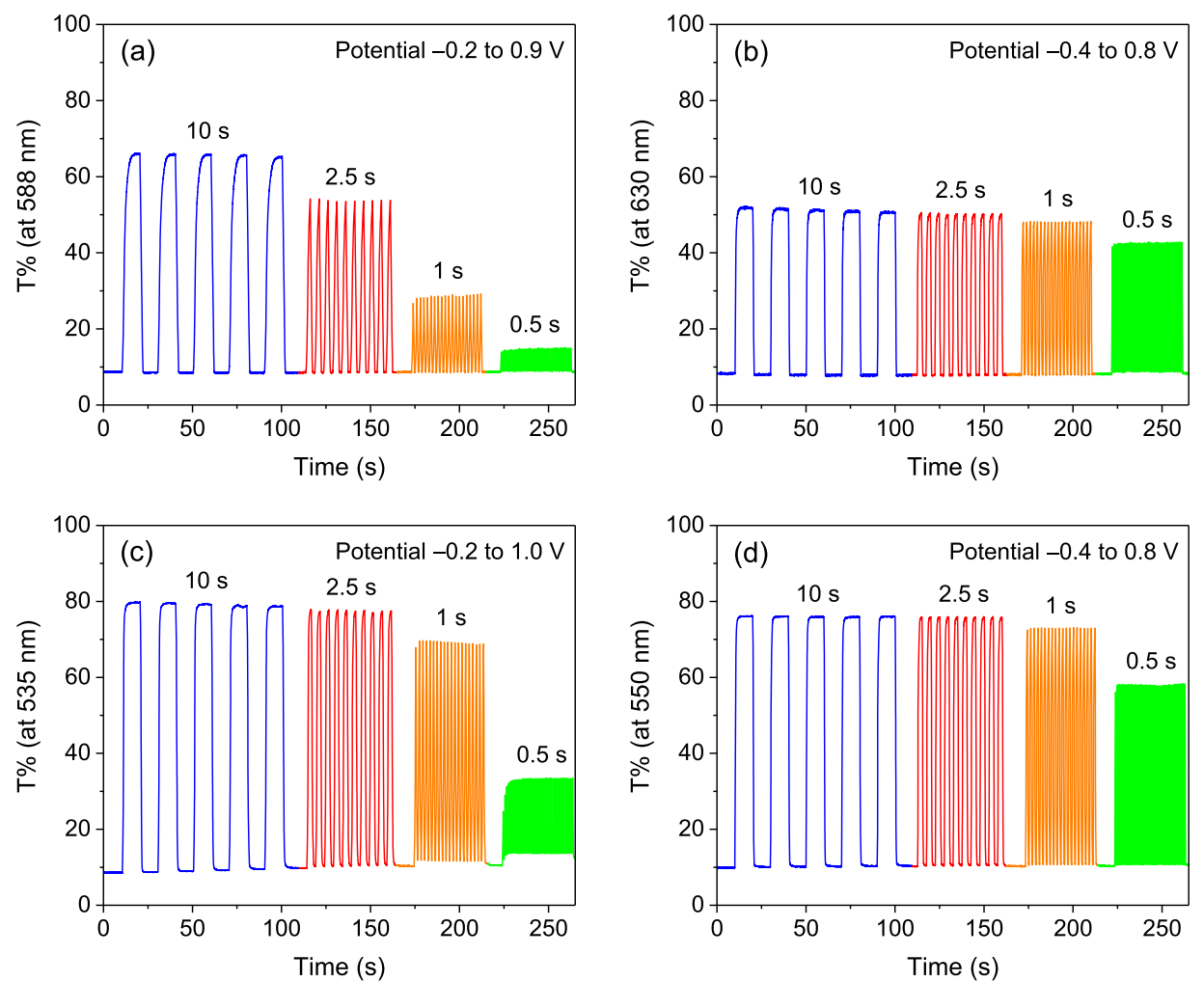

Figure 5. Kinetic measurements of spin-coated (a) PIDTT-TBT, (b) PIDTT-EBE, (c) PIDTT-TBzT, and (d) PIDTT-EBzE films, as measured at the maximum transmittance difference in the electrochromic spectra with potentials $v s \mathrm{Fc} / \mathrm{Fc}^{+}$. The film thickness was $190 \mathrm{~nm}$ for PIDTT-TBT, $150 \mathrm{~nm}$ for PIDTT-EBE, $90 \mathrm{~nm}$ for PIDTT-TBzT, and $115 \mathrm{~nm}$ for PIDTT-EBzE. The supporting electrolyte was $0.1 \mathrm{M}$ solution of $\mathrm{NBu}_{4} \mathrm{PF}_{6}$ in anhydrous acetonitrile.

and the resulting spectra of the spin-coated films are shown in Figure 4 and those of the spray-coated films are included in Figure S14 (Supporting Information). The electrochromic characterization was performed by a stepwise increase in the applied potential at intervals of $0.1 \mathrm{~V}$ in the range from -0.4 to $1.0 \mathrm{~V}$. The blue line represents the absorption of the neutral polymer at $-0.4 \mathrm{~V}$ through the black lines at increasing potentials to the red line describing the absorption of the fully oxidized polymer at $1.0 \mathrm{~V}$. Both spin- and spray-coated polymers featured spectra that are closely similar to the absorption profiles shown in Figure 1, with minor discrepancies in the relative peak intensities, which we attribute to the slightly different stacking of the polymer chains on the ITOglass substrate as compared to the neat polymer films on the glass substrate. Note that the electrochromic spectra are reported in situ after applying two electrochemical redox cycles in the supporting electrolyte (see the Supporting Information for details).

The strong absorption of PIDTT-TBT, PIDTT-EBE, PIDTT-TBzT, and PIDTT-EBzE is fully bleached and a new absorption band in the NIR region is formed upon oxidation in all cases. The newly formed absorption band red-shifted and eventually fell out of the measured spectral range at $1.0 \mathrm{~V}$, although the broad absorption of PIDTT-TBT diminished already at $0.9 \mathrm{~V}$ and that of PIDTT-EBE diminished at a slightly lower potential of $0.8 \mathrm{~V}$. Higher applied potentials did not deliver any observable benefit in further bleaching the visible absorption of these two polymers. In fact, the spraycoated films showed signs of excess doping taking place at 1.0 $\mathrm{V}$, which is regarded as overoxidation and observed as an emerging blue absorption band at 400-500 nm (see Figure
S14a,b, Supporting Information). The spin-coated films featured superior stability toward high voltages. In the case of PIDTT-TBzT, the absorption is rapidly extinguished between 0.4 and $0.8 \mathrm{~V}$ but the far-red/NIR tail of the oxidized polymer kept bleaching up to $1.0 \mathrm{~V}$ in both spin- and spraycoated films, producing fully transparent states. Sufficient bleaching of PIDTT-EBzE is obtained by stepwise oxidation to $0.8 \mathrm{~V}$, without any sign of overoxidation at higher potentials.

Color coordinates of the polymer films in the Commission Internationale de l'Eclairage (CIE) $L^{*} a^{*} b^{*}$ color space are included in Figure S15 and Table S3 (Supporting Information), whereas the observed colors in the red-green-blue (RGB) color space are visualized by the transmission ( $T \%$ ) spectra in Figures S16 and S17 (Supporting Information) and further illustrated by the photographs therein. As expected, PIDTT-TBT displayed a color-neutral, strongly absorbent dark gray/black neutral state. The green tint of PIDTT-EBE originates from the small transmission window in the 525-575 $\mathrm{nm}$ spectral region. PIDTT-TBzT and PIDTT-EBzE featured deep-pink and purple neutral states, respectively. Despite the distinctively different optical absorptions of the four polymers, they all featured similarly bleached oxidized states with the minor difference that the PIDTT-EBE/ PIDTT-EBzE pair showed a relatively stronger residual absorption tail in the far-red/NIR region as compared to the PIDTT-TBT/PIDTT-TBzT pair, causing relatively more blue transmission in the former two polymers (see Figures S16 and S17, Supporting Information). This observation is in good agreement with our earlier discussion about Figure 1 because the stronger donor contribution in the optical absorption of EDOT-containing polymers translates to a stronger polaronic 
band in Figures 4 and S14 (Supporting Information), as compared to the weaker thiophene donor-containing polymers (see ref 76 for the detailed constitution of polaronic and bipolaronic absorption). We note that the coloration and bleaching of the spray-coated films were almost identical to those of the spin-coated films, which is further observed from the superimposed color coordinates following the same bleaching pattern in Figure S15 (Supporting Information). The electrochromic performance of the polymers reported in this work is sufficiently robust regardless of the coating method, thus allowing facile scale up of electrochromic applications.

The speed of optical switching between fully oxidized and reduced states, that is, kinetics, of the spin-coated polymer films is shown in Figure 5 and that of the spray-coated films is included in Figure S18 (Supporting Information). The switching kinetics were measured at the absorption maxima of PIDTT-TBT, PIDTT-EBE, PIDTT-TBzT, and PIDTTEBzE, as specified in the figures. The potential range was chosen for each polymer based on the full $\mathrm{CV}$ redox cycle in Figure S12 (Supporting Information) and the achieved bleaching in Figures 4 and S14 (Supporting Information). The oxidation and reduction were recorded at constant potential steps over $10,2.5,1$, and $0.5 \mathrm{~s}$.

We observe that the full optical switching capacity of PIDTT-TBT is steadily decreased at increasing speed from 10 $\mathrm{s}$ to $2.5 \mathrm{~s}$ and faster, the maximum speed for full switch laying in the $5 \mathrm{~s}$ timeframe. For the other three polymers, PIDTTEBE, PIDTT-TBzT, and PIDTT-EBzE, the performance is maintained at $2.5 \mathrm{~s}$ and close to that at even higher speeds. Only PIDTT-TBzT showed a significant delay in $0.5 \mathrm{~s}$ switching (observed as shift of the baseline in Figure 5c), which we tentatively attribute to the low polarity of the polymer backbone that made it susceptible for charge trapping, in line with the limited electrochemical stability of the polymer (see Figures S11a,b and S12c, Supporting Information). This assumption is further supported by the fact that the less-polar PIDTT-TBT/PIDTT-TBzT pair exhibited faster switching kinetics when spray-coated, while the more polar PIDTT$\mathrm{EBE} / \mathrm{PIDTT}-\mathrm{EBzE}$ pair performed faster by spin coating (see Figures 5 and S18, Supporting Information). Spray-coated polymer films tend to exhibit a rougher morphology than their spin-coated counterparts, and the spray-coated films may accordingly facilitate better ingress of the electrolyte ions for improved charge transport and stabilization of the oxidized polymer. $^{60,64,77,78}$ This is observed as improved kinetics, particularly in the case of the spray-coated PIDTT-TBzT film. The same is true for PIDTT-TBT, which, however, did not show any obvious optical sign of charge trapping neither in the spin-coated nor spray-coated films. In the case of PIDTT$\mathrm{EBE}$ and PIDTT-EBzE, spin-coated films were more effective in facilitating a polar environment for charge transport on one hand and efficient electrochemical oxidation and reduction of the polymer backbone on the other hand.

The promising electrochromic performance of the polymers encouraged us to further study their long-term kinetics, and the measurements were carried out for the spin-coated polymer films over 1800 redox cycles. A switching speed of $5 \mathrm{~s}$ was selected for PIDTT-TBT to obtain full oxidation and reduction but $2.5 \mathrm{~s}$ was enough for PIDTT-EBE, PIDTTTBzT, and PIDTT-EBzE because of their fast kinetics, corresponding to $300 \mathrm{~min}$ and $150 \mathrm{~min}$ of uninterrupted operation. The acetonitrile-based supporting electrolyte was replaced by the corresponding propylene carbonate solution to prevent evaporation of the solvent during these long experiments (acetonitrile bp $82{ }^{\circ} \mathrm{C}$ vs propylene carbonate bp $240{ }^{\circ} \mathrm{C}$ ). The potential range of the polymers is well within the electrochemical window of these solvents. Figure S19 (Supporting Information) shows that all four polymers featured good long-term switching stabilities. PIDTT-TBT, PIDTT-EBE, and PIDTT-EBzE maintained $>90 \%$ of their maximum optical contrast $(\Delta T \%)$ after 1800 redox cycles (i.e., 3600 switches). However, PIDTT-TBzT was an exception in that the polymer featured a slow decrease in optical contrast up to $c$ a. 1000 cycles, after which the performance stabilized and remained essentially constant ( $80 \%$ of $\Delta T \%$ retained). We attribute the initial conditioning of PIDTT-TBzT to the somewhat limited electrochemical stability and related charge trapping in the spin-coated film, as discussed above. To get further insights into this hypothesis, we carried out electrochemical impedance spectroscopy (EIS) measurements for the spin-coated polymer films before and after the long-term kinetic measurements. The EIS curves were recorded at an increasing applied potential, as detailed in Figure S20 (Supporting Information). The general observation is that the resistance of the films was not significantly decreased when the potential was increased above $0.8 \mathrm{~V}$ (for PIDTT-TBT and PIDTT-TBzT) and $0.4 \mathrm{~V}$ (for PIDTT-EBE and PIDTT$\mathrm{EBzE}$ ), thus further confirming that the polymer chains are fully oxidized and the films are saturated at their corresponding bleaching potentials (see Figure 4). The decreasing resistance in the order PIDTT-TBzT > PIDTT-TBT > PIDTT-EBzE $>$ PIDTT-EBE is well in line with the (designed) increasing polarity of the polymers and the (observed) improving electrochemical stability of the spin-coated films, specifically, in the same order. The EIS curves of PIDTT-TBT, PIDTTEBE, and PIDTT-EBzE remained unchanged after 1800 redox cycles, which further demonstrates the good long-term stability of the films and the suitability of the polymers for real-life electrochromic applications.

The ability of the polymers to retain their bleached state and store the injected charge was studied by fully oxidizing the spin-coated films and recording the development of the optical absorption every $30 \mathrm{~s}$ over a period of $300 \mathrm{~s}$, before finally discharging the films electrochemically. The resulting spectra are shown in Figure S21 (Supporting Information). All four polymers featured diminishingly small self-discharging in situ (i.e., immersed in the electrolyte), which is observed as maintained bleached states after switching off the electrical circuit. The PIDTT-TBT/PIDTT-TBzT pair showed almost complete recovery of the optical absorption after electrochemical discharging. From the PIDTT-EBE/PIDTT-EBzE pair, the absorption of PIDTT-EBE was completely recovered, while PIDTT-EBzE displayed a decreased optical density by ca. 0.2 a.u. (corresponding to a $20 \%$ decrease). We attribute the incomplete optical regeneration of PIDTT-EBzE to leaching of the well-maintained oxidized polymer film from the ITO-glass surface instead of incomplete discharging because the discharged film (Figure S21d, Supporting Information) lacks the characteristic $>800 \mathrm{~nm}$ absorption band of a partially bleached polymer (see Figure $4 \mathrm{~d}$ ). The leaching would be overcome in solid-state devices, where the photoactive layer is sandwiched between two electrodes. ${ }^{6,12}$ An essential feature of electrochromic polymers is that they can be used in passive displays and other optoelectronic applications more energy efficiently, if they are able to maintain their 

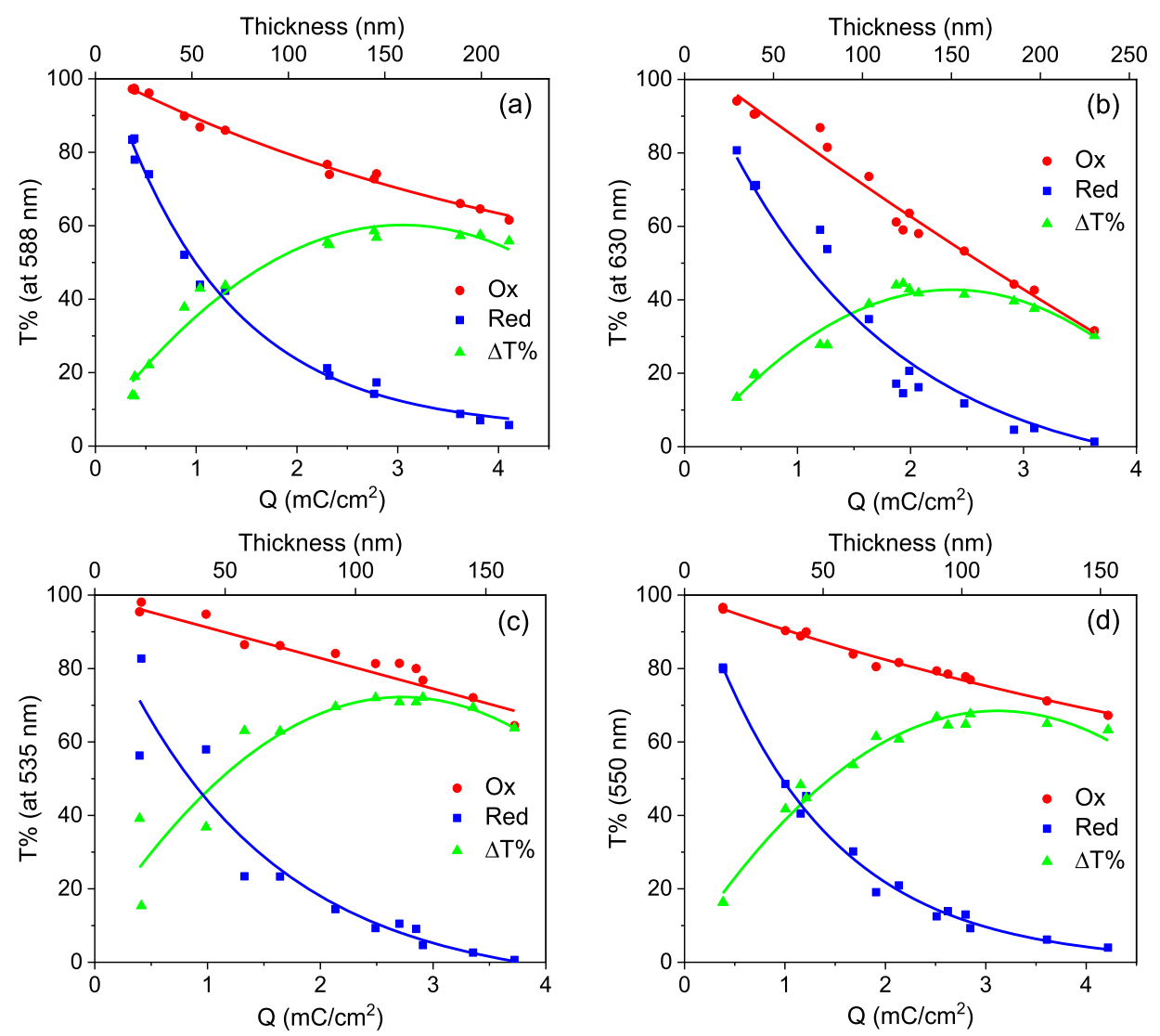

Figure 6. Transmittance of spin-coated (a) PIDTT-TBT, (b) PIDTT-EBE, (c) PIDTT-TBzT, and (d) PIDTT-EBzE films as a function of redox charge density and film thickness (symbols) with the curve fits (lines). Optical contrast (green) is calculated as the transmittance difference between the oxidized (red) and reduced (blue) states. The supporting electrolyte was $0.1 \mathrm{M}$ solution of $\mathrm{NBu}_{4} \mathrm{PF}_{6}$ in anhydrous acetonitrile.

Table 1. Summary of the Performance of Spin- and Spray-Coated Polymer Films ${ }^{a}$

\begin{tabular}{|c|c|c|c|c|c|c|c|c|c|}
\hline \multirow[b]{2}{*}{ polymer } & \multicolumn{2}{|c|}{$\begin{array}{c}\text { maximum } \\
\text { contrast } \\
(\Delta T \%)\end{array}$} & \multicolumn{2}{|c|}{$\begin{array}{c}\text { redox charge } \\
\text { density } \\
(Q \mathrm{mC} \\
\left.\mathrm{cm}^{-2}\right)\end{array}$} & \multicolumn{2}{|c|}{$\begin{array}{l}\text { coloration } \\
\text { efficiency } \\
\left(\eta, \mathrm{cm}^{2} \mathrm{C}^{-1}\right)\end{array}$} & \multirow{2}{*}{$\frac{\begin{array}{c}\text { volumetric charge } \\
\left(Q_{v}, \mathrm{mC}^{-3}\right)\end{array}}{\text { spin }}$} & \multirow{2}{*}{$\begin{array}{c}\begin{array}{c}\text { volumetric capacitance } \\
\left(C_{v}, \mathrm{mF} \mathrm{cm}^{-3}\right)\end{array} \\
\text { spin }\end{array}$} & \multirow{2}{*}{$\frac{\begin{array}{c}\text { specific capacitance } \\
\left(C_{s}, \mathrm{~F} \mathrm{~g}^{-1}\right)\end{array}}{\text { spin }}$} \\
\hline & spin & spray & spin & spray & spin & spray & & & \\
\hline PIDTT-TBT & 60 & 55 & 3.1 & 3.2 & 250 & 220 & $1.9[2.1 \pm 0.2] \times 10^{5}$ & $1.7[1.9 \pm 0.1] \times 10^{5}$ & 150 \\
\hline PIDTT-EBE & 43 & 35 & 2.4 & 2.4 & 230 & 130 & $1.6[1.8 \pm 0.4] \times 10^{5}$ & $1.3[1.5 \pm 0.4] \times 10^{5}$ & 110 \\
\hline PIDTT-TBzT & 72 & 66 & 2.7 & 2.7 & 360 & 330 & $2.3[2.6 \pm 0.6] \times 10^{5}$ & $1.9[2.2 \pm 0.5] \times 10^{5}$ & 160 \\
\hline PIDTT-EBzE & 69 & 60 & 3.1 & 3.2 & 300 & 260 & $2.8[2.7 \pm 0.5] \times 10^{5}$ & $2.3[2.2 \pm 0.4] \times 10^{5}$ & 190 \\
\hline
\end{tabular}

${ }^{a}$ The values are estimated from the fits in Figures 6 and S22 (Supporting Information) at their peak optical contrasts, while those in brackets are averaged over at least 10 different samples with the film thickness varying in the full range of Figure $6 a-d$.

colored and bleached states without an external bias, as successfully demonstrated for the four polymers in this work. An interesting observation outside the presented data is that all four polymers maintained their bleached state also when exposed to ambient air outside the supporting electrolyte for $30 \mathrm{~min}$ or so, and the colored state was recovered by applying a $\mathrm{CV}$ redox cycle back in the electrolyte solution.

Figure 6 shows the evolution of the optical contrast as a function of film thickness and redox charge density $(Q)$ for the spin-coated polymers, while the corresponding data for spraycoated polymers are included in Figure S22 (Supporting Information). Linear correlation was found between the film thickness and $Q$. Table 1 summarizes the electrochromic performance and the charge consumed-or stored-in the process of obtaining the reported $\Delta T \%$ values, as calculated from integrated $\mathrm{CV}$ redox cycles at $100 \mathrm{mV} \mathrm{s}^{-1}$ in the corresponding anodic potential range (see the Supporting Information for details). For the volumetric charge $\left(Q_{v}\right)$ and volumetric capacitance $\left(C_{\mathrm{v}}\right)$ describing the available redox cites per volume, we focus on spin-coated films because the high surface roughness of spray-coated films would translate to a relatively large error in the measured thickness.

The optical contrast was systematically higher for the spincoated films in all four polymers, with a notion that the lesspolar PIDTT-TBT and PIDTT-TBzT featured higher $\Delta T \%$ than the corresponding more polar PIDTT-EBE and PIDTT-EBzE, respectively. Another general observation is that PIDTT-TBT and PIDTT-EBE reached their $\Delta T \%$ at a film thickness of $c a .150 \mathrm{~nm}$ but in the case of PIDTT-TBzT and PIDTT-EBzE, the maximum optical contrast was achieved with slightly thinner films of $c a .110 \mathrm{~nm}$ (see Figure 6 , lines), as expected from the higher absorption coefficients of 
the latter two polymers. Contrary to $\Delta T \%$, the redox charge density was far less sensitive to the film quality, and both spinand spray-coated films exhibited almost identical charge densities within $\pm 0.1 \mathrm{mC} \mathrm{cm}{ }^{-2}$ deviation (see Table 1). The coloration efficiency $(\eta)$ was in good agreement with the observed $\Delta T \%$ for both spin- and spray-coated films, and the higher optical contrast of spin-coated films translated to higher $\eta$, as summarized in Table 1 . To the best of our knowledge, these values rank to the top of all-polymer electrochromic supercapacitors $^{38,48,63}$ and among the best-performing blackto-transparent and colored-to-transparent electrochromic polymers. $^{27,33,64}$ The record $\eta$ values are more than three times higher for conventional electrochromic polymers, which stem from their approximately three times lower charge densities compared to the IDTT-based polymers in this study. $^{79}$

Efficient doping/dedoping of PIDTT-TBT and PIDTTEBzE (illustrated by the XPS atomic composition of the oxidized/neutral films in Table S2, Supporting Information) is observed as the highest $Q$ among the four polymers, whereas the less efficiently dedoped PIDTT-EBE and PIDTT-TBzT featured lower charge densities. A further observation is that the thinner films of PIDTT-TBzT and PIDTT-EBzE led to higher $Q_{\mathrm{v}}$ and $C_{\mathrm{v}}$ as compared to the thicker PIDTT-TBT and PIDTT-EBE films (Table 1). The measured $Q_{\mathrm{v}}$ and $C_{\mathrm{v}}$ correlate well with the optical contrast of the four polymers, and the low contrast of PIDTT-EBE is inherently linked to its incomplete dedoping and lowest $C_{\mathrm{v}}$ in this series. PIDTTEBzE stands out, as it featured a highest $C_{\mathrm{v}}$ of $2.3 \times 10^{5} \mathrm{mF}$ $\mathrm{cm}^{-3}$, which we attribute to the strong polymer-electrolyte interaction and sufficient stabilization of the charged states following the complete doping/dedoping process. This is further justified by comparing the scan rate dependency of the capacitance of PIDTT-EBzE in acetonitrile and propylene carbonate electrolytes (Figure S23a,c, Supporting Information). The $C_{\mathrm{v}}$ was significantly higher in the acetonitrile electrolyte at all scan rates, eventually exceeding $3 \times 10^{5} \mathrm{mF}$ $\mathrm{cm}^{-3}$ at $5 \mathrm{mV} \mathrm{s}^{-1}$, thanks to the enhanced ion ingress. The corresponding $\mathrm{CV}$ redox cycles became relatively more resistive at high scan rates without a significant change in the peak currents above $500 \mathrm{mV} \mathrm{s}^{-1}$, as the ions could not get sufficient time to diffuse into the films (see Figure S23b,d, Supporting Information). We note, however, that the polymer featured excellent electrochemical stability even under the latter conditions, where it could not be stabilized by the electrolyte ions. Assuming an average density of $c a .1 .2 \mathrm{~g} \mathrm{~cm}^{-3}$ of a conjugated polymer (thiophene- and EDOT-based, alkyl side chains containing polymers typically ranging between 1.0 and $\left.1.4 \mathrm{~g} \mathrm{~cm}^{-3}\right),{ }^{70,80}$ the $C_{\mathrm{v}}$ of PIDTT-TBT, PIDTT-EBE, PIDTT-TBzT, and PIDTT-EBzE measured at the high scan rate of $100 \mathrm{mV} \mathrm{s}^{-1}$ corresponds to a $C_{\mathrm{s}}$ of approximately 150 , 110,160 , and $190 \mathrm{~F} \mathrm{~g} \mathrm{~g}^{-1}$, respectively, which are the highest values reported for all-polymer electrochromic supercapacitors (for comparison, see Table S4 and Figure S24, Supporting Information). ${ }^{38,46,48,63}$ It is then significant that the $C_{s}$ of PIDTT-EBzE reaches $260 \mathrm{~F} \mathrm{~g}^{-1}$ at the low scan rate of $5 \mathrm{mV}$ $\mathrm{s}^{-1}$ and compares to that of the state-of-the-art polymer composite electrodes used in supercapacitors-as a single electro-optical material. $^{21,41,50}$

Overall, we attribute the high charge storage capacity to the combination of (i) strong absorption of the polymers that allows coating of the films at low thickness and (ii) effective stabilization of the oxidized polymers by the optimized electrolyte that facilitates (iii) substantially high doping levels and full charging and discharging within a 5-10 s timeframe, thereby making PIDTT-TBT, PIDTT-EBE, PIDTT-TBzT, and PIDTT-EBzE an intriguing set of polymers with regard to hybrid electrochromic and energy storage applications. Moreover, IDTT-based polymers are promising anodic (p-type) electrodes that can be used to develop electrochromic asymmetric supercapacitors with a wide potential window, in combination with cathodic (n-type) polymer electrodes, so as to obtain very high-energy density devices.

\section{CONCLUSIONS}

This work represented the fusion of electrochromic and energy-storing donor-acceptor copolymers. The optical and electrochemical properties of the polymers were varied by copolymerizing the IDTT donor monomer with four different DAD segments, based either on a stronger electron acceptor in PIDTT-TBT and PIDTT-EBE or a weaker electron acceptor in PIDTT-TBzT and PIDTT-EBzE. The acceptors were endowed either with the more polar EDOT or less-polar thiophene spacers. The polymers featured strong optical absorption but their distinctively different colors were attributed to the DAD units and changes in the ICT character in the polymer backbone. The absorption of PIDTT-TBT spanned over the entire visible spectrum and produced an achromatic dark gray/black color, while the spectrum of PIDTT-EBE extended further into the NIR region with a maintained absorption coefficient and green tint to the film. The coalescence of the two-band absorption of PIDTT-TBT and PIDTT-EBE into a narrow single band in PIDTT-TBzT and PIDTT-EBzE due to a weak ICT interaction is observed as a deep-pink and purple color, respectively. Despite the different colorations, all four polymers were effectively oxidized to produce similarly bleached transparent states. The IDTT structure stabilized the doped states and allowed electrochemical switching within a low potential range of $\Delta V \leq 1.2 \mathrm{~V}$. Hence, the polymers showed excellent long-term switching stabilities, which is manifested by maintaining $80-98 \%$ of their initial optical contrast after 1800 charge-discharge cycles. PIDTT-EBzE outperformed the other polymers in this study, as the strong polymer-electrolyte interaction, separation of the polymer chains by alkyl side chains, and efficient electrochemical doping (and dedoping) of the polar backbone in the excess of $100 \%$ had a direct impact in delivering the highest specific capacitance out of the four polymers, reaching $260 \mathrm{~F}$ $\mathrm{g}^{-1}$ at $5 \mathrm{mV} \mathrm{s}^{-1}$. Nevertheless, the capacitances of the polymers were all in the range of $110-190 \mathrm{~F} \mathrm{~g}^{-1}$ at $100 \mathrm{mV} \mathrm{s}^{-1}$, which rank as the highest values reported for all-polymer electrochromic supercapacitors. It is significant that the IDTT-based polymers deliver an electro-optical performance that is close to that of the state-of-the-art nanostructured composite electrodes but importantly allow much simplified solution-based fabrication of electrochromic supercapacitors. Further optimization is needed to surpass the performance of the composite electrodes, for example, by improving the electrolyte ion transport in well-structured polymer architectures. This work provides a new approach not only to (i) the design of electrochemically stable alternatives to the EDOT-based polymers aiming at high-contrast electrochromic switching and easy color tuning but also to (ii) the development of new types of energy-storing polymers that exhibit high capacitances and enable fast charging and discharging on demand and their synergistic applications in color-indicating and self-regulating 
smart energy systems that can visually show the stored energy level and, if integrated with OPVs, simultaneously harvest and store solar energy.

\section{ASSOCIATED CONTENT}

\section{SI Supporting Information}

The Supporting Information is available free of charge at https://pubs.acs.org/doi/10.1021/acs.macromol.0c02212.

Material characterization, synthesis, TGA and DSC, density functional theory calculations, cyclic voltammetry, X-ray photoelectron spectroscopy, electrochromic characterization, electrochemical impedance spectroscopy, comparison of hybrid electrochromic and energy storage polymers (PDF)

\section{AUTHOR INFORMATION}

\section{Corresponding Authors}

Mats R. Andersson - Flinders Institute for Nanoscale Science and Technology, Flinders University, Adelaide, South Australia 5042, Australia; (1) orcid.org/0000-0002-79288216; Email: mats.andersson@flinders.edu.au

Ergang Wang - Department of Chemistry and Chemical Engineering/Applied Chemistry, Chalmers University of Technology, Gothenburg SE-412 96, Sweden; School of Materials Science and Engineering, Zhengzhou University, Zhengzhou 450001, China; 이이이.org/0000-0002-49423771; Email: ergang@chalmers.se

\section{Authors}

Petri Murto - Department of Chemistry and Chemical Engineering/Applied Chemistry, Chalmers University of Technology, Gothenburg SE-412 96, Sweden; Flinders Institute for Nanoscale Science and Technology, Flinders University, Adelaide, South Australia 5042, Australia; Department of Chemistry, University of Cambridge, Cambridge CB2 1EW, United Kingdom; ○ orcid.org/00000001-7618-000X

Sait Elmas - Flinders Institute for Nanoscale Science and Technology, Flinders University, Adelaide, South Australia 5042, Australia; (1) orcid.org/0000-0002-1235-1436

Ulises A. Méndez-Romero - Department of Chemistry and Chemical Engineering/Applied Chemistry, Chalmers University of Technology, Gothenburg SE-412 96, Sweden; Centro de Investigación en Materiales Avanzados S.C. (CIMAV), Apodaca, Nuevo León 66628, Mexico

Yanting Yin - Flinders Institute for Nanoscale Science and Technology, Flinders University, Adelaide, South Australia 5042, Australia

Zewdneh Genene - Department of Chemistry and Chemical Engineering/Applied Chemistry, Chalmers University of Technology, Gothenburg SE-412 96, Sweden

Mariza Mone - Department of Chemistry and Chemical Engineering/Applied Chemistry, Chalmers University of Technology, Gothenburg SE-412 96, Sweden; Flinders Institute for Nanoscale Science and Technology, Flinders University, Adelaide, South Australia 5042, Australia

Gunther G. Andersson - Flinders Institute for Nanoscale Science and Technology, Flinders University, Adelaide, South Australia 5042, Australia; (1) orcid.org/0000-0001-57423037

Complete contact information is available at: https://pubs.acs.org/10.1021/acs.macromol.0c02212

\section{Author Contributions}

P.M. and S.E. contributed equally to this work.

\section{Notes}

The authors declare no competing financial interest.

\section{ACKNOWLEDGMENTS}

We thank Dr. Andreas Dahlin and Oliver Olsson at the Chalmers University of Technology for their help with defining the color coordinates. We thank the Swedish Research Council (2015-04853, 2016-06146, 2019-02345, and 2019-04683), the Swedish Research Council Formas, and the Wallenberg Foundation (2017.0186, 2016.0059) for financial support. P.M. acknowledges funding from the Emil Aaltonen Foundation. S.E. and M.R.A. thank the Australian Government through the Australian Research Council's Discovery Projects funding scheme (project DP160102356) and the Defence Innovation Partnership (DIP).

\section{REFERENCES}

(1) Beaujuge, P. M.; Reynolds, J. R. Color Control in $\pi$-Conjugated Organic Polymers for Use in Electrochromic Devices. Chem. Rev. 2010, 110, 268-320.

(2) Gunbas, G.; Toppare, L. Electrochromic conjugated polyheterocycles and derivatives-highlights from the last decade towards realization of long lived aspirations. Chem. Commun. 2012, 48, 10831101.

(3) Neo, W. T.; Ye, Q.; Chua, S.-J.; Xu, J. Conjugated polymer-based electrochromics: materials, device fabrication and application prospects. J. Mater. Chem. C 2016, 4, 7364-7376.

(4) Lv, X.; Li, W.; Ouyang, M.; Zhang, Y.; Wright, D. S.; Zhang, C. Polymeric electrochromic materials with donor-acceptor structures. J. Mater. Chem. C 2017, 5, 12-28.

(5) Yang, G.; Zhang, Y.-M.; Cai, Y.; Yang, B.; Gu, C.; Zhang, S. X.-A. Advances in nanomaterials for electrochromic devices. Chem. Soc. Rev. 2020, DOI: 10.1039/D0CS00317D.

(6) Bella, F.; Leftheriotis, G.; Griffini, G.; Syrrokostas, G.; Turri, S.; Grätzel, M.; Gerbaldi, C. A New Design Paradigm for Smart Windows: Photocurable Polymers for Quasi-Solid Photoelectrochromic Devices with Excellent Long-Term Stability under Real Outdoor Operating Conditions. Adv. Funct. Mater. 2016, 26, 1127-1137.

(7) Wang, Y.; Runnerstrom, E. L.; Milliron, D. J. Switchable Materials for Smart Windows. Annu. Rev. Chem. Biomol. Eng. 2016, 7, 283-304.

(8) Kim, J.; Rémond, M.; Kim, D.; Jang, H.; Kim, E. Electrochromic Conjugated Polymers for Multifunctional Smart Windows with Integrative Functionalities. Adv. Mater. Technol. 2020, 5, 1900890.

(9) Österholm, A. M.; Shen, D. E.; Kerszulis, J. A.; Bulloch, R. H.; Kuepfert, M.; Dyer, A. L.; Reynolds, J. R. Four Shades of Brown: Tuning of Electrochromic Polymer Blends Toward High-Contrast Eyewear. ACS Appl. Mater. Interfaces 2015, 7, 1413-1421.

(10) Remmele, J.; Shen, D. E.; Mustonen, T.; Fruehauf, N. High Performance and Long-Term Stability in Ambiently Fabricated Segmented Solid-State Polymer Electrochromic Displays. ACS Appl. Mater. Interfaces 2015, 7, 12001-12008.

(11) Lang, A. W.; Österholm, A. M.; Reynolds, J. R. Paper-Based Electrochromic Devices Enabled by Nanocellulose-Coated Substrates. Adv. Funct. Mater. 2019, 29, 1903487.

(12) Jensen, J.; Hösel, M.; Dyer, A. L.; Krebs, F. C. Development and Manufacture of Polymer-Based Electrochromic Devices. Adv. Funct. Mater. 2015, 25, 2073-2090.

(13) Li, X.; Perera, K.; He, J.; Gumyusenge, A.; Mei, J. Solutionprocessable electrochromic materials and devices: roadblocks and strategies towards large-scale applications. J. Mater. Chem. C 2019, 7, 12761-12789.

(14) Heeger, A. J. Charge Storage in Conducting Polymers: Solitons, Polarons, and Bipolarons. Polym. J. 1985, 17, 201-208. 
(15) Rughooputh, S. D. D. V.; Nowak, M.; Hotta, S.; Heeger, A. J.; Wudl, F. Soluble conducting polymers: The poly(3-alkylthienylenes). Synth. Met. 1987, 21, 41-50.

(16) Nowak, M. J.; Speigel, D.; Hotta, S.; Heeger, A. J.; Pincus, P. A. Charge storage on a conducting polymer in solution. Synth. Met. $1989,28,399-406$.

(17) Kim, J.; Lee, J.; You, J.; Park, M.-S.; Hossain, M. S. A.; Yamauchi, Y.; Kim, J. H. Conductive polymers for next-generation energy storage systems: recent progress and new functions. Mater. Horiz. 2016, 3, 517-535.

(18) Dubal, D. P.; Chodankar, N. R.; Kim, D.-H.; Gomez-Romero, P. Towards flexible solid-state supercapacitors for smart and wearable electronics. Chem. Soc. Rev. 2018, 47, 2065-2129.

(19) Zhang, Z.; Liao, M.; Lou, H.; Hu, Y.; Sun, X.; Peng, H. Conjugated Polymers for Flexible Energy Harvesting and Storage. Adv. Mater. 2018, 30, 1704261.

(20) Guo, Q.; Zhao, X.; Li, Z.; Wang, B.; Wang, D.; Nie, G. High Performance Multicolor Intelligent Supercapacitor and Its Quantitative Monitoring of Energy Storage Level by Electrochromic Parameters. ACS Appl. Energy Mater. 2020, 3, 2727-2736.

(21) Zhao, C.; Jia, X.; Shu, K.; Yu, C.; Wallace, G. G.; Wang, C. Conducting polymer composites for unconventional solid-state supercapacitors. J. Mater. Chem. A 2020, 8, 4677-4699.

(22) Cho, J.; Yun, T. Y.; Noh, H. Y.; Baek, S. H.; Nam, M.; Kim, B.; Moon, H. C.; Ko, D. H. Semitransparent Energy-Storing Functional Photovoltaics Monolithically Integrated with Electrochromic Supercapacitors. Adv. Funct. Mater. 2020, 30, 1909601.

(23) Dyer, A. L.; Thompson, E. J.; Reynolds, J. R. Completing the Color Palette with Spray-Processable Polymer Electrochromics. ACS Appl. Mater. Interfaces 2011, 3, 1787-1795.

(24) Kerszulis, J. A.; Johnson, K. E.; Kuepfert, M.; Khoshabo, D.; Dyer, A. L.; Reynolds, J. R. Tuning the painter's palette: subtle steric effects on spectra and colour in conjugated electrochromic polymers. J. Mater. Chem. C 2015, 3, 3211-3218.

(25) Cao, K.; Shen, D. E.; Österholm, A. M.; Kerszulis, J. A.; Reynolds, J. R. Tuning Color, Contrast, and Redox Stability in High Gap Cathodically Coloring Electrochromic Polymers. Macromolecules 2016, 49, 8498-8507.

(26) Teran, N. B.; Reynolds, J. R. Discrete Donor-Acceptor Conjugated Systems in Neutral and Oxidized States: Implications toward Molecular Design for High Contrast Electrochromics. Chem. Mater. 2017, 29, 1290-1301.

(27) Lo, C. K.; Shen, D. E.; Reynolds, J. R. Fine-Tuning the Color Hue of $\pi$-Conjugated Black-to-Clear Electrochromic Random Copolymers. Macromolecules 2019, 52, 6773-6779.

(28) Poverenov, E.; Zamoshchik, N.; Patra, A.; Ridelman, Y.; Bendikov, M. Unusual Doping of Donor-Acceptor-Type Conjugated Polymers Using Lewis Acids. J. Am. Chem. Soc. 2014, 136, 51385149.

(29) Zeglio, E.; Vagin, M.; Musumeci, C.; Ajjan, F. N.; Gabrielsson, R.; Trinh, X. T.; Son, N. T.; Maziz, A.; Solin, N.; Inganäs, O. Conjugated Polyelectrolyte Blends for Electrochromic and Electrochemical Transistor Devices. Chem. Mater. 2015, 27, 6385-6393.

(30) Ming, S.; Zhen, S.; Lin, K.; Zhao, L.; Xu, J.; Lu, B. Thiadiazolo[3,4-c]pyridine as an Acceptor toward Fast-Switching Green Donor-Acceptor-Type Electrochromic Polymer with Low Bandgap. ACS Appl. Mater. Interfaces 2015, 7, 11089-11098.

(31) Sassi, M.; Salamone, M. M.; Ruffo, R.; Patriarca, G. E.; Mari, C. M.; Pagani, G. A.; Posset, U.; Beverina, L. State-of-the-Art Neutral Tint Multichromophoric Polymers for High-Contrast See-Through Electrochromic Devices. Adv. Funct. Mater. 2016, 26, 5240-5246.

(32) Yen, H.-J.; Liou, G.-S. Recent advances in triphenylamine-based electrochromic derivatives and polymers. Polym. Chem. 2018, 9, 3001-3018.

(33) Zhang, Q.; Tsai, C.-Y.; Li, L.-J.; Liaw, D.-J. Colorless-to-colorful switching electrochromic polyimides with very high contrast ratio. Nat. Commun. 2019, 10, 1239.

(34) Zhang, H.; Yao, M.; Wei, J.; Zhang, Y.; Zhang, S.; Gao, Y.; Li, J.; Lu, P.; Yang, B.; Ma, Y. Stable p/n-Dopable Conducting Redox
Polymers for High-Voltage Pseudocapacitor Electrode Materials: Structure-Performance Relationship and Detailed Investigation into Charge-Trapping Effect. Adv. Energy Mater. 2017, 7, 1701063.

(35) Horng, Y.-Y.; Lu, Y.-C.; Hsu, Y.-K.; Chen, C.-C.; Chen, L.-C.; Chen, K.-H. Flexible supercapacitor based on polyaniline nanowires/ carbon cloth with both high gravimetric and area-normalized capacitance. J. Power Sources 2010, 195, 4418-4422.

(36) Wang, K.; Zou, W.; Quan, B.; Yu, A.; Wu, H.; Jiang, P.; Wei, Z. An All-Solid-State Flexible Micro-supercapacitor on a Chip. Adv. Energy Mater. 2011, 1, 1068-1072.

(37) Sharma, S.; Soni, R.; Kurungot, S.; Asha, S. K. Naphthalene Diimide Copolymers by Direct Arylation Polycondensation as Highly Stable Supercapacitor Electrode Materials. Macromolecules 2018, 51, 954-965.

(38) Wang, K.; Huang, L.; Eedugurala, N.; Zhang, S.; Sabuj, M. A.; Rai, N.; Gu, X.; Azoulay, J. D.; Ng, T. N. Wide Potential Window Supercapacitors Using Open-Shell Donor-Acceptor Conjugated Polymers with Stable N-Doped States. Adv. Energy Mater. 2019, 9, 1902806.

(39) Liu, W.; Ulaganathan, M.; Abdelwahab, I.; Luo, X.; Chen, Z.; Rong Tan, S. J.; Wang, X.; Liu, Y.; Geng, D.; Bao, Y.; Chen, J.; Loh, K. P. Two-Dimensional Polymer Synthesized via Solid-State Polymerization for High-Performance Supercapacitors. ACS Nano 2018, 12, $852-860$.

(40) Liao, Y.; Wang, H.; Zhu, M.; Thomas, A. Efficient Supercapacitor Energy Storage Using Conjugated Microporous Polymer Networks Synthesized from Buchwald-Hartwig Coupling. Adv. Mater. 2018, 30, 1705710.

(41) Chen, X.; Lin, H.; Chen, P.; Guan, G.; Deng, J.; Peng, H. Smart, Stretchable Supercapacitors. Adv. Mater. 2014, 26, 4444-4449.

(42) Snook, G. A.; Kao, P.; Best, A. S. Conducting-polymer-based supercapacitor devices and electrodes. J. Power Sources 2011, 196, 112.

(43) Österholm, A. M.; Shen, D. E.; Dyer, A. L.; Reynolds, J. R. Optimization of PEDOT Films in Ionic Liquid Supercapacitors: Demonstration As a Power Source for Polymer Electrochromic Devices. ACS Appl. Mater. Interfaces 2013, 5, 13432-13440.

(44) Kim, D.; Zozoulenko, I. Why Is Pristine PEDOT Oxidized to 33\%? A Density Functional Theory Study of Oxidative Polymerization Mechanism. J. Phys. Chem. B 2019, 123, 5160-5167.

(45) Eric Shen, D.; Österholm, A. M.; Reynolds, J. R. Out of sight but not out of mind: the role of counter electrodes in polymer-based solid-state electrochromic devices. J. Mater. Chem. C 2015, 3, 97159725.

(46) Guo, Y.; Li, W.; Yu, H.; Perepichka, D. F.; Meng, H. Flexible Asymmetric Supercapacitors via Spray Coating of a New Electrochromic Donor-Acceptor Polymer. Adv. Energy Mater. 2017, 7, 1601623.

(47) Ponder, J. F.; Österholm, A. M.; Reynolds, J. R. Conjugated Polyelectrolytes as Water Processable Precursors to Aqueous Compatible Redox Active Polymers for Diverse Applications: Electrochromism, Charge Storage, and Biocompatible Organic Electronics. Chem. Mater. 2017, 29, 4385-4392.

(48) Ming, S.; Li, Z.; Zhen, S.; Liu, P.; Jiang, F.; Nie, G.; Xu, J. Highperformance D-A-D type electrochromic polymer with $\pi$ spacer applied in supercapacitor. Chem. Eng. J. 2020, 390, 124572.

(49) Sun, Y.; Zhu, G.; Zhao, X.; Kang, W.; Li, M.; Zhang, X.; Yang, H.; Guo, L.; Lin, B. Solution-processable, hypercrosslinked polymer via post-crosslinking for electrochromic supercapacitor with outstanding electrochemical stability. Sol. Energy Mater. Sol. Cells 2020, 215,110661 .

(50) Yun, T. G.; Park, M.; Kim, D.-H.; Kim, D.; Cheong, J. Y.; Bae, J. G.; Han, S. M.; Kim, I.-D. All-Transparent Stretchable Electrochromic Supercapacitor Wearable Patch Device. ACS Nano 2019, 13, $3141-3150$

(51) Xu, X.; Cai, P.; Lu, Y.; Choon, N. S.; Chen, J.; Ong, B. S.; Hu, $X$. Synthesis of a Novel Low-Bandgap Polymer Based on a LadderType Heptacyclic Arene Consisting of Outer Thieno[3,2-b]thiophene 
Units for Efficient Photovoltaic Application. Macromol. Rapid Commun. 2013, 34, 681-688.

(52) Dang, D.; Chen, W.; Himmelberger, S.; Tao, Q.; Lundin, A.; Yang, R.; Zhu, W.; Salleo, A.; Müller, C.; Wang, E. Enhanced Photovoltaic Performance of Indacenodithiophene-Quinoxaline Copolymers by Side-Chain Modulation. Adv. Energy Mater. 2014, 4, 1400680.

(53) Xu, X.; Li, Z.; Bäcke, O.; Bini, K.; James, D. I.; Olsson, E.; Andersson, M. R.; Wang, E. Effects of Side Chain Isomerism on the Physical and Photovoltaic Properties of Indacenodithieno[3,2-b]thiophene-Quinoxaline Copolymers: Toward a Side Chain Design for Enhanced Photovoltaic Performance. J. Mater. Chem. A 2014, 2, 18988-18997.

(54) Benavides, C. M.; Murto, P.; Chochos, C. L.; Gregoriou, V. G.; Avgeropoulos, A.; Xu, X.; Bini, K.; Sharma, A.; Andersson, M. R.; Schmidt, O.; Brabec, C. J.; Wang, E.; Tedde, S. F. High-Performance Organic Photodetectors from a High-Bandgap IndacenodithiopheneBased $\pi$-Conjugated Donor-Acceptor Polymer. ACS Appl. Mater. Interfaces 2018, 10, 12937-12946.

(55) Murto, P.; Genene, Z.; Benavides, C. M.; Xu, X.; Sharma, A.; Pan, X.; Schmidt, O.; Brabec, C. J.; Andersson, M. R.; Tedde, S. F.; Mammo, W.; Wang, E. High Performance All-Polymer Photodetector Comprising a Donor-Acceptor-Acceptor Structured Indacenodithiophene-Bithieno[3,4-c]Pyrroletetrone Copolymer. ACS Macro Lett. 2018, 7, 395-400.

(56) Zhang, W.; Han, Y.; Zhu, X.; Fei, Z.; Feng, Y.; Treat, N. D.; Faber, H.; Stingelin, N.; McCulloch, I.; Anthopoulos, T. D.; Heeney, M. A Novel Alkylated Indacenodithieno[3,2-b]thiophene-Based Polymer for High-Performance Field-Effect Transistors. Adv. Mater. 2016, 28, 3922-3927.

(57) Barłóg, M.; Zhang, X.; Kulai, I.; Yang, D. S.; Sredojevic, D. N.; Sil, A.; Ji, X.; Salih, K. S. M.; Bazzi, H. S.; Bronstein, H.; Fang, L.; Kim, J.; Marks, T. J.; Guo, X.; Al-Hashimi, M. Indacenodithiazole-LadderType Bridged Di(thiophene)-Difluoro-Benzothiadiazole-Conjugated Copolymers as Ambipolar Organic Field-Effect Transistors. Chem. Mater. 2019, 31, 9488-9496.

(58) Minotto, A.; Murto, P.; Genene, Z.; Zampetti, A.; Carnicella, G.; Mammo, W.; Andersson, M. R.; Wang, E.; Cacialli, F. Efficient Near-Infrared Electroluminescence at $840 \mathrm{~nm}$ with "Metal-Free" Small-Molecule:Polymer Blends. Adv. Mater. 2018, 30, 1706584.

(59) Murto, P.; Tang, S.; Larsen, C.; Xu, X.; Sandström, A.; Pietarinen, J.; Bagemihl, B.; Abdulahi, B. A.; Mammo, W.; Andersson, M. R.; Wang, E.; Edman, L. Incorporation of Designed DonorAcceptor-Donor Segments in a Host Polymer for Strong NearInfrared Emission from a Large-Area Light-Emitting Electrochemical Cell. ACS Appl. Energy Mater. 2018, 1, 1753-1761.

(60) Tang, S.; Murto, P.; Wang, J.; Larsen, C.; Andersson, M. R.; Wang, E.; Edman, L. On the Design of Host-Guest Light-Emitting Electrochemical Cells: Should the Guest be Physically Blended or Chemically Incorporated into the Host for Efficient Emission? Adv. Opt. Mater. 2019, 7, 1900451.

(61) Cheng, X.; Ju, X.; Du, H.; Zhang, Y.; Zhao, J.; Xie, Y. Synthesis and characterization of novel donor-acceptor type electrochromic polymers containing diketopyrrolopyrrole as an acceptor and propylenedioxythiophene or indacenodithiophene as a donor. RSC Adv. 2018, 8, 23119-23129.

(62) Bini, K.; Murto, P.; Elmas, S.; Andersson, M. R.; Wang, E. Broad spectrum absorption and low-voltage electrochromic operation from indacenodithieno[3,2-b]thiophene-based copolymers. Polym. Chem. 2019, 10, 2004-2014.

(63) Sun, Y.; Zhao, X.; Zhu, G.; Li, M.; Zhang, X.; Yang, H.; Lin, B. Twisted ladder-like donor-acceptor polymers as electrode materials for flexible electrochromic supercapacitors. Electrochim. Acta 2020, 333, 135495.

(64) Padilla, J.; Österholm, A. M.; Dyer, A. L.; Reynolds, J. R. Process controlled performance for soluble electrochromic polymers. Sol. Energy Mater. Sol. Cells 2015, 140, 54-60.
(65) Yuksel, R.; Cevher, S. C.; Cirpan, A.; Toppare, L.; Unalan, H. E. All-Organic Electrochromic Supercapacitor Electrodes. J. Electrochem. Soc. 2015, 162, A2805-A2810.

(66) Li, W.; Guo, Y.; Wang, Y.; Xing, X.; Chen, X.; Ning, J.; Yu, H.; Shi, Y.; Murtaza, I.; Meng, H. A "chain-lock" strategy to construct a conjugated copolymer network for supercapacitor applications. $J$. Mater. Chem. A 2019, 7, 116-123.

(67) Beaujuge, P. M.; Ellinger, S.; Reynolds, J. R. Spray Processable Green to Highly Transmissive Electrochromics via Chemically Polymerizable Donor-Acceptor Heterocyclic Pentamers. Adv. Mater. 2008, 20, 2772-2776.

(68) Vangerven, T.; Verstappen, P.; Drijkoningen, J.; Dierckx, W.; Himmelberger, S.; Salleo, A.; Vanderzande, D.; Maes, W.; Manca, J. V. Molar Mass versus Polymer Solar Cell Performance: Highlighting the Role of Homocouplings. Chem. Mater. 2015, 27, 3726-3732.

(69) Brebels, J.; Klider, K. C. C. W. S.; Kelchtermans, M.; Verstappen, P.; Van Landeghem, M.; Van Doorslaer, S.; Goovaerts, E.; Garcia, J. R.; Manca, J.; Lutsen, L.; Vanderzande, D.; Maes, W. Low bandgap polymers based on bay-annulated indigo for organic photovoltaics: Enhanced sustainability in material design and solar cell fabrication. Org. Electron. 2017, 50, 264-272.

(70) Beaujuge, P. M.; Amb, C. M.; Reynolds, J. R. Spectral Engineering in $\pi$-Conjugated Polymers with Intramolecular DonorAcceptor Interactions. Acc. Chem. Res. 2010, 43, 1396-1407.

(71) Chan, S.-H.; Chen, C.-P.; Chao, T.-C.; Ting, C.; Lin, C.-S.; Ko, B.-T. Synthesis, Characterization, and Photovoltaic Properties of Novel Semiconducting Polymers with Thiophene-PhenyleneThiophene (TPT) as Coplanar Units. Macromolecules 2008, 41, 5519-5526.

(72) Xu, Y.-X.; Chueh, C.-C.; Yip, H.-L.; Chang, C.-Y.; Liang, P.-W.; Intemann, J. J.; Chen, W.-C.; Jen, A. K.-Y. Indacenodithieno[3,2b]thiophene-based broad bandgap polymers for high efficiency polymer solar cells. Polym. Chem. 2013, 4, 5220-5223.

(73) Hillman, A. R.; Daisley, S. J.; Bruckenstein, S. Ion and solvent transfers and trapping phenomena during n-doping of PEDOT films. Electrochim. Acta 2008, 53, 3763-3771.

(74) Heinze, J.; Frontana-Uribe, B. A.; Ludwigs, S. Electrochemistry of Conducting Polymers-Persistent Models and New Concepts. Chem. Rev. 2010, 110, 4724-4771.

(75) Rajapakse, R. M. G.; Attanayake, N. H.; Karunathilaka, D.; Steen, A. E.; Hammer, N. I.; Strongin, D. R.; Watkins, D. L. Advances in electro-copolymerization of NIR emitting and electronically conducting block copolymers. J. Mater. Chem. C 2019, 7, 3168-3172.

(76) Zozoulenko, I.; Singh, A.; Singh, S. K.; Gueskine, V.; Crispin, X.; Berggren, M. Polarons, Bipolarons, And Absorption Spectroscopy of PEDOT. ACS Appl. Polym. Mater. 2019, 1, 83-94.

(77) Mortimer, R. J.; Graham, K. R.; Grenier, C. R. G.; Reynolds, J. $\mathrm{R}$. Influence of the Film Thickness and Morphology on the Colorimetric Properties of Spray-Coated Electrochromic Disubstituted 3,4-Propylenedioxythiophene Polymers. ACS Appl. Mater. Interfaces 2009, 1, 2269-2276.

(78) Sandström, A.; Asadpoordarvish, A.; Enevold, J.; Edman, L. Spraying Light: Ambient-Air Fabrication of Large-Area Emissive Devices on Complex-Shaped Surfaces. Adv. Mater. 2014, 26, 49754980.

(79) Reeves, B. D.; Grenier, C. R. G.; Argun, A. A.; Cirpan, A.; McCarley, T. D.; Reynolds, J. R. Spray Coatable Electrochromic Dioxythiophene Polymers with High Coloration Efficiencies. Macromolecules 2004, 37, 7559-7569.

(80) Sonmez, G.; Meng, H.; Wudl, F. Very Stable Low Band Gap Polymer for Charge Storage Purposes and Near-Infrared Applications. Chem. Mater. 2003, 15, 4923-4929. 